All102 74 1666

A UNITED STATES

DEPARTMENT OF

COMMERCE

PUBLICATION

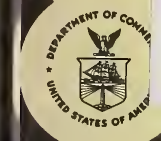

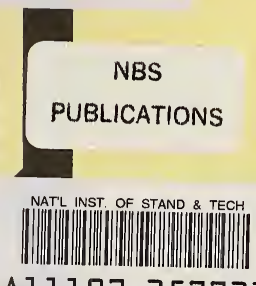

Al1107 257721
NBS SPECIAL PUBLICATION

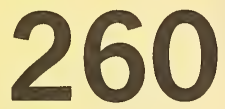

SUPPLEMENT

\title{
Standard Reference Materials
}

U.S.
DEPARTMENT
OF
COMMERCE
National
Bureau
of
Standards
Q6
100
.457
1973
$54 p 91$.
6.2




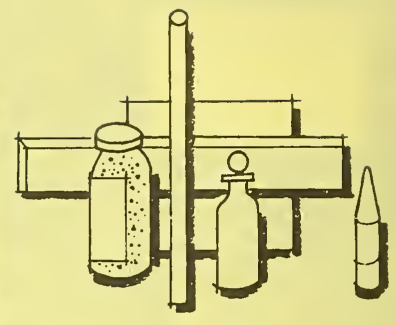

Standard Reference Materials are wellcharacterized materials dissemenated by the National Bureau of Standards to be used to calibrate and evaluate measuring instruments, methods, and systems or to produce scientific data that can be referred readily to a common base. The materials are certified for chemical composition or for a particular physical or chemical property. They are used on-site in science and industry to calibrate instruments and methods used in production and quality control of raw materials, chemicals, metals, ceramics, fuels, and radioactive nuclides in manufacturing processes and in research. 


\section{ORDERING}

\section{GENERAL}

Orders should be addressed to the Office of Standard Reference Materials, National Bureau of Standards, Washington, D.C. 20234. Telephonic or telegraphic communications should be addressed to the attention of the Office of Standard Reference Materials, (Telephone 301-921-2045). Orders should give the amount (number of units), catalog number and name of the standard requested. For example: $150 \mathrm{~g}$ (1 unit) of No. $11 \mathrm{~h}$ Basic-Open-Hearth Steel, 0.2 percent C. These materials are distributed only in the units listed.

Acceptance of an order does not imply acceptance of any provision set forth in the order contrary to the policy, practice, or regulations of the National Bureau of Standards or the U.S. Government. Prices listed herein are subject to change without notice. Price changes when made are first announced in various NBS publications, especially the Technical News Bulletin, and in announcements mailed to users of these materials.

Prices in effect at time of shipment will be billed to the purchaser. No discounts are given on NBS Standard Reference Materials.

To provide better service to users of SRM's our name-label files are periodically updated and/or corrected. If your name and address are not correct, please return the name-label portion of the envelope and indicate the corrections. Send it and all other inquiries to:

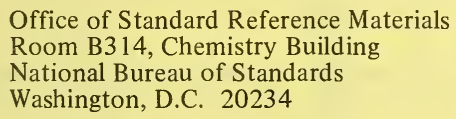

\section{FOREIGN ORDERS}

A. Prepaid orders will be processed, subject to export-import regulations of the United States and the country from which the order originates, and shipped within 5 days provided export or import license is not required. (See mode of shipment-- Foreign Shipments.) Prepayment may be made by any of the following:

1. UNESCO coupons;

2. Bankers' draft against U.S. bank;

3. Bank to bank transfer on U.S. bank;

4. Letter of credit on a U.S. bank;

5. International Money Order.

All checks, coupons, etc., should be made payable to the National Bureau of Standards and must be in U.S. dollars.

B. Non-prepaid purchase orders from customers with established credit will be processed within 10 days. Variations in prices and quantities shipped will be noted on invoices. Upon receipt of goods, payment can be made by any of the methods listed under A.

C. Pro-forma service, subject to export-import regulations, may require 60 days or more for processing. Customers are urged to use method A or B whenever possible for fast service and to supply all necessary import documents and information with their order. Payment may be by any of the means shown under $\mathrm{A}$ above. 


\section{DOMESTIC SHIPMENTS}

Shipments of material (except for certain restricted categories, e.g., hydrocarbons, organic sulfur compounds, special nuclear materials, compressed gases and radioactive standards) intended for the United States, Mexico, and Canada are normally shipped prepaid air parcel post (providing that the parcel does not exceed the weight limits as prescribed by Postal Laws and Regulations) unless the purchaser requests a different mode of shipment, in which case the shipment will be sent collect. It is impractical for the Bureau to prepay shipping charges and add this cost to the billing invoice. Hydrocarbons, organic sulfur compounds, compressed gases, rubber compounding materials, radioactive standards and similar materials are shipped express collect.

\section{FOREIGN SHIPMENTS}

Both prepaid and non-prepaid orders will be shipped by prepaid International Air Parcel Post, subject to size, weight, and category of material limitations. Any other mode of shipment requested by customer must be paid for by the customer. Shipments excluded from International Air Parcel Post for any reason, must be handled through an agent (shipping or brokerage firm) located in the U.S. as designated by the purchaser. These parcels will be packed for overseas shipment and forwarded via express collect to the U.S. firm designated as agent. NOTE: Nuclear SRM's will not be shipped through an agent, they will be shipped direct.

\section{TECHNICAL INQUIRIES}

All technical inquiries regarding SRM's, RM's, and GM's should be directed to the Office of Standard Reference Materials, National Bureau of Standards, Washington, D.C. 20234. Telephone (301) 921-2045. 


\section{SECTION I}

AVAILABILITY* AND PRICE LIST

\section{A. STANDARD REFERENCE MATERIALS}

*SRM's listed in italics are in preparation.

\begin{tabular}{|c|c|c|c|c|c|c|c|}
\hline SRM & Type & Unit & Price & SRM & Type & Unit & Price \\
\hline $\begin{array}{l}1 b \\
3 b \\
4 j \\
5 \mathrm{~L} \\
6 \mathrm{~g}\end{array}$ & 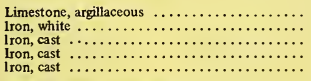 & $\begin{array}{r}50 \mathrm{~g} \\
110 \mathrm{~g} \\
150 \mathrm{~g} \\
150 \mathrm{~g} \\
150 \mathrm{~g}\end{array}$ & $\begin{array}{l}36 \\
37 \\
37 \\
45 \\
40\end{array}$ & $\begin{array}{l}123 \mathrm{c} \\
124 \mathrm{~d} \\
125 \mathrm{~b} \\
126 \mathrm{c} \\
127 \mathrm{~b}\end{array}$ & 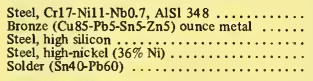 & $\begin{array}{l}150 \mathrm{~g} \\
150 \mathrm{~g} \\
150 \mathrm{~g} \\
150 \mathrm{~g} \\
150 \mathrm{~g}\end{array}$ & $\begin{array}{r}\$ 37 \\
37 \\
37 \\
37 \\
37\end{array}$ \\
\hline $\begin{array}{r}7 \mathrm{~g} \\
8 \mathrm{j} \\
10 \mathrm{~g} \\
11 \mathrm{~h} \\
12 \mathrm{~h}\end{array}$ & 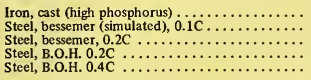 & $\begin{array}{l}150 \mathrm{~g} \\
150 \mathrm{~g} \\
150 \mathrm{~g} \\
150 \mathrm{~g} \\
150 \mathrm{~g}\end{array}$ & $\begin{array}{l}37 \\
37 \\
37 \\
37 \\
37\end{array}$ & $\begin{array}{l}129 \mathrm{c} \\
131 \mathrm{~b} \\
132 \mathrm{~b} \\
133 \mathrm{a} \\
134 \mathrm{a}\end{array}$ & 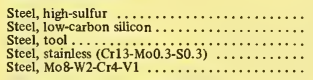 & $\begin{array}{l}150 \mathrm{~g} \\
100 \mathrm{~g} \\
150 \mathrm{~g} \\
150 \mathrm{~g} \\
150 \mathrm{~g}\end{array}$ & $\begin{array}{l}37 \\
31 \\
37 \\
37 \\
37\end{array}$ \\
\hline $\begin{array}{l}13 \mathrm{~g} \\
14 \mathrm{e} \\
15 \mathrm{~g} \\
16 \mathrm{e} \\
17\end{array}$ & 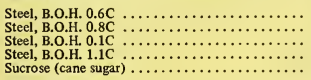 & $\begin{array}{r}150 \mathrm{~g} \\
150 \mathrm{~g} \\
150 \mathrm{~g} \\
150 \mathrm{~g} \\
60 \mathrm{~g}\end{array}$ & $\begin{array}{l}37 \\
37 \\
37 \\
37 \\
30\end{array}$ & $\begin{array}{l}136 \mathrm{c} \\
139 \mathrm{a} \\
140 \mathrm{~b} \\
141 \mathrm{~b} \\
142\end{array}$ & 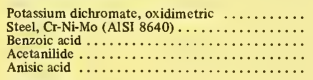 & $\begin{array}{r}60 \mathrm{~g} \\
150 \mathrm{~g} \\
2 \mathrm{~g} \\
2 \mathrm{~g} \\
2 \mathrm{~g}\end{array}$ & $\begin{array}{l}36 \\
37 \\
32 \\
32 \\
30\end{array}$ \\
\hline $\begin{array}{l}19 \mathrm{~g} \\
20 \mathrm{~g} \\
25 \mathrm{c} \\
27 \mathrm{e} \\
30 \mathrm{f}\end{array}$ & 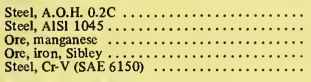 & $\begin{array}{l}150 \mathrm{~g} \\
150 \mathrm{~g} \\
100 \mathrm{~g} \\
100 \mathrm{~g} \\
150 \mathrm{~g}\end{array}$ & $\begin{array}{l}37 \\
37 \\
31 \\
32 \\
37\end{array}$ & $\begin{array}{l}143 \mathrm{~b} \\
147 \\
148 \\
152 \mathrm{a} \\
153 \mathrm{a}\end{array}$ & 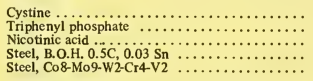 & $\begin{array}{r}2 \mathrm{~g} \\
2 \mathrm{~g} \\
2 \mathrm{~g} \\
150 \mathrm{~g} \\
150 \mathrm{~g}\end{array}$ & $\begin{array}{l}33 \\
32 \\
28 \\
37 \\
37\end{array}$ \\
\hline $\begin{array}{l}32 \mathrm{e} \\
33 \mathrm{~d} \\
36 \mathrm{~b} \\
37 \mathrm{e} \\
39 \mathrm{i}\end{array}$ & 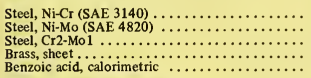 & $\begin{array}{r}150 \mathrm{~g} \\
150 \mathrm{~g} \\
150 \mathrm{~g} \\
150 \mathrm{~g} \\
30 \mathrm{~g}\end{array}$ & $\begin{array}{l}37 \\
37 \\
37 \\
37 \\
36\end{array}$ & $\begin{array}{l}154 \mathrm{~b} \\
155 \\
157 \mathrm{a} \\
158 \mathrm{a} \\
160 \mathrm{~b}\end{array}$ & 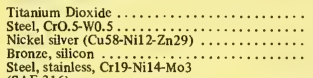 & $\begin{array}{r}90 \mathrm{~g} \\
150 \mathrm{~g} \\
135 \mathrm{~g} \\
150 \mathrm{~g}\end{array}$ & $\begin{array}{l}54 \\
37 \\
37 \\
37\end{array}$ \\
\hline $\begin{array}{l}40 \mathrm{~h} \\
41 \mathrm{a} \\
42 \mathrm{~g} \\
43 \mathrm{~h} \\
44 \mathrm{f} \\
45 \mathrm{~d}\end{array}$ & 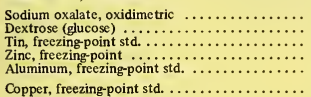 & $\begin{array}{r}60 \mathrm{~g} \\
70 \mathrm{~g} \\
350 \mathrm{~g} \\
350 \mathrm{~g} \\
200 \mathrm{~g} \\
450 \mathrm{~g}\end{array}$ & $\begin{array}{l}36 \\
30 \\
60 \\
50 \\
75 \\
50\end{array}$ & $\begin{array}{l}162 a \\
163 \\
166 \mathrm{c} \\
168 \\
171\end{array}$ & 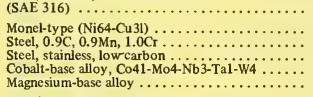 & $\begin{array}{l}150 \mathrm{~g} \\
150 \mathrm{~g} \\
100 \mathrm{~g} \\
100 \mathrm{~g} \\
150 \mathrm{~g} \\
100 \mathrm{~g}\end{array}$ & $\begin{array}{l}37 \\
37 \\
44 \\
29 \\
37 \\
37\end{array}$ \\
\hline $\begin{array}{l}49 \mathrm{e} \\
50 \mathrm{c} \\
51 \mathrm{~b} \\
53 \mathrm{e} \\
54 \mathrm{~d}\end{array}$ & 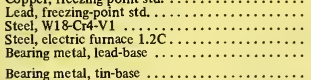 & $\begin{array}{l}600 \mathrm{~g} \\
150 \mathrm{~g} \\
150 \mathrm{~g} \\
150 \mathrm{~g} \\
170 \mathrm{~g}\end{array}$ & $\begin{array}{l}50 \\
37 \\
37 \\
37 \\
37\end{array}$ & $\begin{array}{l}173 \mathrm{a} \\
174 \\
176 \\
178 \\
180\end{array}$ & 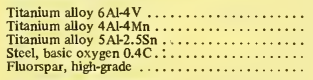 & $\begin{array}{l}100 \mathrm{~g} \\
100 \mathrm{~g} \\
100 \mathrm{~g} \\
150 \mathrm{~g} \\
120 \mathrm{~g}\end{array}$ & $\begin{array}{l}37 \\
37 \\
37 \\
37 \\
44\end{array}$ \\
\hline $\begin{array}{l}54 \mathrm{a} \\
55 \mathrm{e} \\
57 \\
58 \mathrm{a} \\
59 \mathrm{a} \\
64 \mathrm{~b}\end{array}$ & 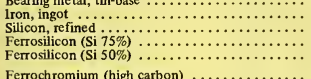 & $\begin{array}{r}150 \mathrm{~g} \\
60 \mathrm{~g} \\
75 \mathrm{~g} \\
50 \mathrm{~g}\end{array}$ & $\begin{array}{l}37 \\
33 \\
50 \\
44 \\
35\end{array}$ & $\begin{array}{l}181 \\
182 \\
183 \\
184 \\
185 \mathrm{e}\end{array}$ & $\begin{array}{l}\text { Ore, lithium (Spodumene) } \ldots \ldots \ldots \ldots \ldots \ldots \ldots \ldots \\
\text { Ore, lithium (Petalite) } \ldots \ldots \ldots \ldots \ldots \ldots \ldots \ldots \ldots \\
\text { Ore, lithium (Lepidolite) } \ldots \ldots \ldots \ldots \ldots \ldots \ldots \ldots \\
\text { Bronze, leaded-tin } \ldots \ldots \ldots \ldots \ldots \ldots \\
\text { Potassium hydrogen phthalate, pH } \ldots \ldots \ldots \ldots \ldots\end{array}$ & $\begin{array}{r}45 \mathrm{~g} \\
45 \mathrm{~g} \\
45 \mathrm{~g} \\
150 \mathrm{~g} \\
60 \mathrm{~g}\end{array}$ & $\begin{array}{l}31 \\
31 \\
31 \\
37 \\
39\end{array}$ \\
\hline $\begin{array}{l}64 \mathrm{~b} \\
65 \mathrm{~d} \\
69 \mathrm{a} \\
70 \mathrm{a} \\
71\end{array}$ & 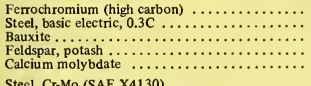 & $\begin{array}{r}100 \mathrm{~g} \\
150 \mathrm{~g} \\
50 \mathrm{~g} \\
40 \mathrm{~g} \\
60 \mathrm{~g} \\
150 \mathrm{~g}\end{array}$ & $\begin{array}{l}35 \\
37 \\
31 \\
36 \\
33 \\
37\end{array}$ & $\begin{array}{l}186 \mathrm{Ic} \\
1861 \mathrm{Ic} \\
187 \mathrm{~b} \\
188 \\
189\end{array}$ & 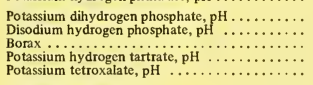 & $\begin{array}{l}30 \mathrm{~g} \\
30 \mathrm{~g} \\
30 \mathrm{~g} \\
60 \mathrm{~g} \\
65 \mathrm{~g}\end{array}$ & $\begin{array}{l}39 \\
34 \\
34 \\
34 \\
34\end{array}$ \\
\hline $\begin{array}{l}72 \mathrm{f} \\
73 \mathrm{c} \\
76 a \\
77 a \\
78 a \\
79 a\end{array}$ & 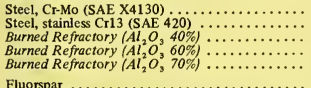 & $\begin{array}{l}150 \mathrm{~g} \\
150 \mathrm{~g}\end{array}$ & $\begin{array}{l}37 \\
37\end{array}$ & $\begin{array}{l}189 \\
191 \\
192 \\
193 \\
194 \\
195\end{array}$ & $\begin{array}{l}\text { Sodium bicarbonate, } \mathrm{pH}, \ldots \ldots \ldots \ldots \ldots \ldots \ldots \\
\text { Sodium carbonate, pH } \ldots \ldots \ldots \ldots \ldots \ldots \\
\text { Potassium Nitrate, Fertilizer } \ldots \ldots \ldots \ldots \ldots \ldots \\
\text { Ammonium dihydrogen phosphate, Fertilizer } \ldots \\
\text { Ferrosilicon }(75 \% \mathrm{Si} \text {, High Purity) } \ldots \ldots \ldots \ldots \ldots\end{array}$ & $\begin{array}{l}30 \mathrm{~g} \\
30 \mathrm{~g} \\
30 \mathrm{~g} \\
90 \mathrm{~g} \\
90 \mathrm{~g} \\
75 \mathrm{~g}\end{array}$ & $\begin{array}{l}34 \\
37 \\
37 \\
49 \\
49 \\
50\end{array}$ \\
\hline $\begin{array}{l}79 \mathrm{a} \\
82 \mathrm{~b} \\
83 \mathrm{c} \\
84 \mathrm{~h} \\
85 \mathrm{~b} \\
87 \mathrm{a}\end{array}$ & 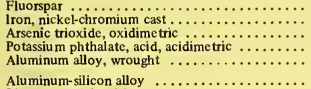 & $\begin{array}{r}120 \mathrm{~g} \\
150 \mathrm{~g} \\
75 \mathrm{~g} \\
60 \mathrm{~g} \\
75 \mathrm{~g} \\
75 \mathrm{~g}\end{array}$ & $\begin{array}{l}44 \\
37 \\
36 \\
30 \\
37 \\
37\end{array}$ & $\begin{array}{l}196 \\
198 \\
199 \\
217 b-5 \\
217 b-8 S\end{array}$ & 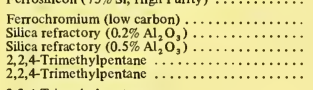 & $\begin{array}{r}100 \mathrm{~g} \\
45 \mathrm{~g} \\
45 \mathrm{~g} \\
5 \mathrm{ml} \\
8 \mathrm{ml}\end{array}$ & $\begin{array}{l}50 \\
49 \\
31 \\
31 \\
62 \\
69\end{array}$ \\
\hline $\begin{array}{l}87 \mathrm{a} \\
88 \mathrm{a} \\
89 \\
90 \\
91 \\
92\end{array}$ & 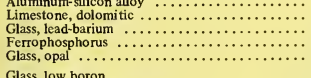 & $\begin{array}{l}13 \mathrm{~g} \\
55 \mathrm{~g} \\
75 \mathrm{~g} \\
45 \mathrm{~g} \\
45\end{array}$ & $\begin{array}{l}37 \\
36 \\
31 \\
33 \\
31 \\
31\end{array}$ & $\begin{array}{l}217 b-25 \\
217 b-50 \\
300 \\
301 \\
302\end{array}$ & 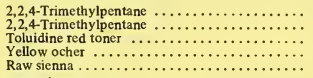 & $\begin{array}{l}25 \mathrm{ml} \\
50 \mathrm{ml} \\
40 \mathrm{~g} \\
45 \mathrm{~g} \\
45 \mathrm{~g}\end{array}$ & $\begin{array}{r}184 \\
334 \\
30 \\
30 \\
30\end{array}$ \\
\hline $\begin{array}{l}92 \\
93 \mathrm{a} \\
94 \mathrm{c} \\
97 \mathrm{a} \\
98 \mathrm{a}\end{array}$ & 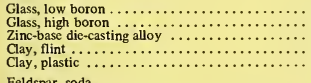 & $\begin{array}{r}45 \mathrm{~g} \\
\mathrm{ea} \\
150 \mathrm{~g} \\
60 \mathrm{~g} \\
60 \mathrm{~g}\end{array}$ & $\begin{array}{l}31 \\
54 \\
37 \\
86 \\
86 \\
36\end{array}$ & $\begin{array}{l}303 \\
304 \\
305 \\
306 \\
307\end{array}$ & 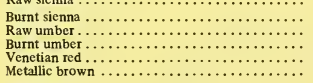 & $\begin{array}{l}45 \mathrm{~g} \\
50 \mathrm{~g} \\
45 \mathrm{~g} \\
50 \mathrm{~g} \\
60 \mathrm{~g} \\
60 \mathrm{~g}\end{array}$ & $\begin{array}{l}30 \\
30 \\
30 \\
30 \\
30 \\
30\end{array}$ \\
\hline $\begin{array}{l}99 \mathrm{a} \\
100 \mathrm{~b} \\
101 \mathrm{f} \\
103 \mathrm{a} \\
104\end{array}$ & 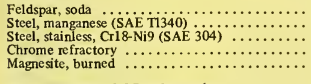 & $\begin{array}{r}40 \mathrm{~g} \\
150 \mathrm{~g} \\
100 \mathrm{~g} \\
60 \mathrm{~g} \\
60 \mathrm{~g}\end{array}$ & $\begin{array}{l}36 \\
37 \\
37 \\
31 \\
31\end{array}$ & $\begin{array}{l}308 \\
309 \\
310 \\
311 \\
312\end{array}$ & 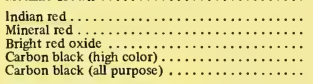 & $\begin{array}{l}50 \mathrm{~g} \\
65 \mathrm{~g} \\
50 \mathrm{~g} \\
10 \mathrm{~g} \\
20 \mathrm{~g}\end{array}$ & $\begin{array}{l}30 \\
30 \\
30 \\
30 \\
30\end{array}$ \\
\hline $\begin{array}{l}105 \\
106 \mathrm{~b} \\
107 \mathrm{~b} \\
112 \\
113 \mathrm{a}\end{array}$ & 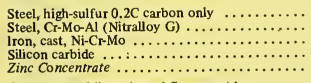 & $\begin{array}{r}150 \mathrm{~g} \\
150 \mathrm{~g} \\
150 \mathrm{~g} \\
85 \mathrm{~g}\end{array}$ & $\begin{array}{l}29 \\
37 \\
37 \\
31\end{array}$ & $\begin{array}{l}313 \\
314 \\
315 \\
316 \\
317\end{array}$ & 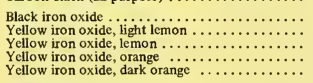 & $\begin{array}{l}42 \mathrm{~g} \\
20 \mathrm{~g} \\
20 \mathrm{~g} \\
25 \mathrm{~g} \\
40 \mathrm{~g}\end{array}$ & $\begin{array}{l}30 \\
30 \\
30 \\
30 \\
30\end{array}$ \\
\hline $\begin{array}{l}114 \mathrm{~L} \\
115 \mathrm{a} \\
120 \mathrm{~b} \\
121 \mathrm{~d} \\
122 \mathrm{e}\end{array}$ & 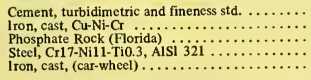 & $\begin{array}{l}\operatorname{set}(20) \\
150 \mathrm{~g} \\
90 \mathrm{~g} \\
150 \mathrm{~g} \\
150 \mathrm{~g}\end{array}$ & $\begin{array}{l}57 \\
37 \\
49 \\
37 \\
37\end{array}$ & $\begin{array}{l}318 \\
319 \\
320 \\
321 \\
322\end{array}$ & 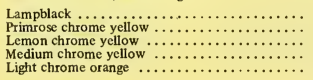 & $\begin{array}{r}15 \mathrm{~g} \\
65 \mathrm{~g} \\
60 \mathrm{~g} \\
65 \mathrm{~g} \\
100 \mathrm{~g}\end{array}$ & $\begin{array}{l}30 \\
30 \\
30 \\
30 \\
30\end{array}$ \\
\hline
\end{tabular}




\begin{tabular}{|c|c|c|c|c|c|c|c|}
\hline SRM & Type & Unit & Price & SRM & Type & Unit & Price \\
\hline $\begin{array}{l}323 \\
324 \\
325 \\
326 \\
327\end{array}$ & 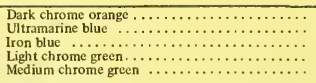 & $\begin{array}{r}100 \mathrm{~g} \\
37 \mathrm{~g} \\
25 \mathrm{~g} \\
60 \mathrm{~g} \\
50 \mathrm{~g}\end{array}$ & $\begin{array}{r}\$ 30 \\
30 \\
30 \\
30 \\
30\end{array}$ & $\begin{array}{l}450 \\
461 \\
462 \\
463 \\
464\end{array}$ & 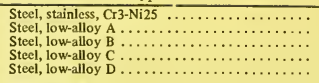 & $\begin{array}{l}\text { ea } \\
\text { ea } \\
\text { ea } \\
\text { ea } \\
\text { ea }\end{array}$ & $\begin{array}{l}\$ 39 \\
39 \\
39 \\
39 \\
39\end{array}$ \\
\hline $\begin{array}{l}328 \\
329 \\
330 \\
331 \\
332\end{array}$ & 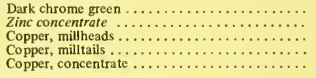 & $\begin{array}{r}45 \mathrm{~g} \\
100 \mathrm{~g} \\
100 \mathrm{~g} \\
50 \mathrm{~g}\end{array}$ & $\begin{array}{l}30 \\
50 \\
50 \\
50\end{array}$ & $\begin{array}{l}465 \\
466 \\
467 \\
468 \\
479\end{array}$ & $\begin{array}{l}\text { Iron, ingot } \mathrm{E} \ldots \ldots \ldots \ldots \ldots \ldots \ldots \\
\text { lron, ingot } \mathrm{F} \ldots \ldots \ldots \ldots \ldots \ldots \ldots \ldots \\
\text { Steel, low-alloy } \mathrm{G} \ldots \ldots \ldots \ldots \ldots \ldots \ldots \\
\text { Steel, low-alloy } \mathrm{H}, \ldots \ldots \ldots \ldots \ldots \ldots \\
\text { Microprobe, F-Cr-Ni Ailoy } \ldots \ldots \ldots \ldots\end{array}$ & $\begin{array}{l}\text { ea } \\
\text { ea } \\
\text { ea } \\
\text { ea } \\
\text { ea }\end{array}$ & $\begin{array}{l}39 \\
39 \\
39 \\
39 \\
54\end{array}$ \\
\hline $\begin{array}{l}333 \\
335 \\
336 \\
337 \\
339\end{array}$ & 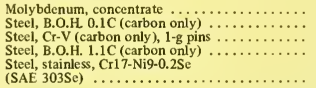 & $\begin{array}{r}35 \mathrm{~g} \\
300 \mathrm{~g} \\
75 \mathrm{~g} \\
300 \mathrm{~g} \\
150 \mathrm{~g}\end{array}$ & $\begin{array}{l}50 \\
31 \\
35 \\
31 \\
44\end{array}$ & $\begin{array}{l}480 \\
481 \\
482 \\
483 \\
485\end{array}$ & 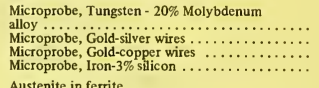 & $\begin{array}{r}\text { ea } \\
\text { set } \\
\text { set } \\
\text { ea }\end{array}$ & $\begin{array}{r}129 \\
134 \\
134 \\
54 \\
80\end{array}$ \\
\hline $\begin{array}{l}340 \\
341 \\
342 \\
342 \mathrm{a} \\
343\end{array}$ & 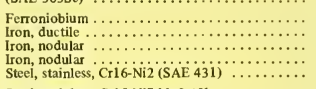 & $\begin{array}{l}100 \mathrm{~g} \\
150 \mathrm{~g} \\
150 \mathrm{~g} \\
150 \mathrm{~g} \\
150 \mathrm{~g}\end{array}$ & $\begin{array}{l}44 \\
49 \\
37 \\
37 \\
39 \\
37\end{array}$ & $\begin{array}{l}485 \\
493 \\
592 \\
593 \\
594 \\
595\end{array}$ & $\begin{array}{l}\text { Austenite in ferrite } \ldots \ldots \ldots \ldots \ldots \ldots \ldots \ldots \\
\text { Iron carbide in ferrite } \ldots \ldots \ldots \ldots \ldots \ldots \\
\text { Hydrocarbon blends }- \text { Biend No. } 1 \ldots \ldots \ldots \ldots \ldots \ldots \\
\text { Hydrocarbon blends }- \text { Blend No. } 2 \ldots \ldots \ldots \ldots \ldots \ldots \\
\text { Hydrocarbon blends - Blend No. } 3 \ldots \ldots \ldots \ldots \ldots\end{array}$ & $\begin{array}{l}\text { ea } \\
\text { ea } \\
\text { set } \\
\text { set } \\
\text { set }\end{array}$ & $\begin{array}{l}89 \\
89 \\
36 \\
36 \\
36\end{array}$ \\
\hline $\begin{array}{l}343 \\
344 \\
345 \\
346 \\
348 \\
349\end{array}$ & 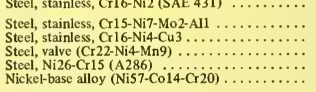 & $\begin{array}{l}150 \mathrm{~g} \\
150 \mathrm{~g} \\
150 \mathrm{~g} \\
150 \mathrm{~g} \\
150 \mathrm{~g} \\
150 \mathrm{~g}\end{array}$ & $\begin{array}{l}37 \\
37 \\
37 \\
44 \\
37 \\
37\end{array}$ & $\begin{array}{l}595 \\
596 \\
597 \\
598 \\
599 \\
607\end{array}$ & $\begin{array}{l}\text { Hydrocarbon blends - Blend No. } 4 \ldots \\
\text { Hydrocarbon blends - Blend No. } 5 \ldots \\
\text { Hydrocarbon blends - Blend No. } 6 \ldots \\
\text { Hydrocarbon blends - Blend No. } 7 \ldots \\
\text { Hydrocarbon blends - Blend No. } 8 \ldots \\
\text { Potassium Feldspar, Trace Rubidium. }\end{array}$ & $\begin{array}{l}\text { set } \\
\text { set } \\
\text { set } \\
\text { set } \\
\text { set }\end{array}$ & $\begin{array}{l}36 \\
36 \\
36 \\
36 \\
36\end{array}$ \\
\hline $\begin{array}{l}350 \\
352 \\
353 \\
354 \\
355\end{array}$ & $\begin{array}{l}\text { Benzoic acid, acidimetric } \ldots \ldots \ldots \ldots \ldots \ldots \ldots \\
\text { Titanium, unalloyed, for hydrogen } \ldots \ldots \ldots \ldots \ldots \\
\text { Titanium, unalloyed, for hydrogen } \ldots \ldots \ldots \ldots \ldots \ldots \\
\text { Titanium, unalloyed, for hydrogen } \ldots \ldots \ldots \ldots \ldots \ldots \\
\text { Titanium, unalloyed, for oxygen } \ldots \ldots \ldots \ldots \ldots \ldots\end{array}$ & $\begin{array}{l}30 \mathrm{~g} \\
20 \mathrm{~g} \\
20 \mathrm{~g} \\
20 \mathrm{~g} \\
20 \mathrm{~g}\end{array}$ & $\begin{array}{l}36 \\
39 \\
39 \\
39 \\
44\end{array}$ & $\begin{array}{l}607 \\
608 \\
609\end{array}$ & $\begin{array}{l}\text { Potassium Feldspar, Trace Rubidium } \\
\text { and Strontium .................... } \\
\text { Glass, trace elements, } \\
\text { set } 1 \text { each } 614 \text { and } 616 \ldots \ldots \ldots \ldots \ldots \\
\text { Glass, trace elements, } \ldots \ldots \ldots \ldots \ldots \\
\text { set } 1 \text { each } 615 \text { and } 617 \ldots \ldots \ldots \ldots\end{array}$ & $\begin{array}{l}5 \mathrm{~g} \\
\text { set } \\
\text { set }\end{array}$ & $\begin{array}{r}40 \\
200 \\
200\end{array}$ \\
\hline $\begin{array}{l}356 \\
360 \mathrm{a} \\
361 \\
362 \\
363\end{array}$ & 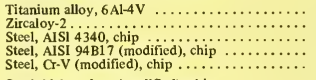 & $\begin{array}{r}20 \mathrm{~g} \\
100 \mathrm{~g} \\
150 \mathrm{~g} \\
150 \mathrm{~g} \\
150 \mathrm{~g}\end{array}$ & $\begin{array}{l}44 \\
59 \\
37 \\
37 \\
37\end{array}$ & $\begin{array}{l}610 \\
611 \\
612 \\
613 \\
614\end{array}$ & 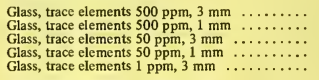 & $\begin{array}{l}\text { ea } \\
\text { ea } \\
\text { ea } \\
\text { ea } \\
\text { ea }\end{array}$ & $\begin{array}{l}67 \\
67 \\
67 \\
67 \\
67\end{array}$ \\
\hline $\begin{array}{l}364 \\
365 \\
366 \\
370 d \\
371 \mathrm{~d}\end{array}$ & 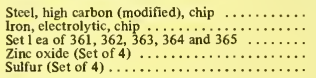 & $\begin{array}{l}150 \mathrm{~g} \\
150 \mathrm{~g} \\
\text { set } \\
8 \mathrm{~kg} \\
6 \mathrm{~kg}\end{array}$ & $\begin{array}{r}37 \\
37 \\
104 \\
38 \\
42\end{array}$ & $\begin{array}{l}615 \\
616 \\
617 \\
618 \\
619\end{array}$ & $\begin{array}{l}\text { Glass, trace elements } 1 \mathrm{ppm}, 1 \mathrm{~mm} \ldots \ldots \ldots \ldots \\
\text { Glass, trace elements } 02 \mathrm{ppm}, 3 \mathrm{~mm} \\
\text { Glass, trace elements } .02 \mathrm{ppm}, 1 \mathrm{~mm} \ldots \ldots \ldots \ldots \ldots \\
\text { Glass, trace elements, } 3 \mathrm{~mm} \ldots \ldots \ldots \ldots \ldots \ldots \ldots \\
\text { Glass, trace elements, } 1 \mathrm{~mm} \ldots \ldots \ldots \ldots \ldots \ldots \ldots\end{array}$ & $\begin{array}{l}\text { ea } \\
\text { ea } \\
\text { ea } \\
\text { set } \\
\text { set }\end{array}$ & $\begin{array}{r}67 \\
67 \\
67 \\
200 \\
200\end{array}$ \\
\hline $\begin{array}{l}372 \mathrm{~g} \\
373 \mathrm{f} \\
374 \mathrm{c} \\
375 \mathrm{f} \\
376 \mathrm{a}\end{array}$ & $\begin{array}{l}\text { Stearic acid (Set of } 4) \\
\text { Benzothiazyl disulfide }(\text { Set of } 4) \ldots \ldots \ldots \ldots \ldots \ldots \\
\text { Tetramethylthiuram disulfide } \ldots \ldots \ldots \ldots \ldots \ldots \ldots \\
\text { Channel black (Set of } 4) \ldots \ldots \ldots \ldots \ldots \ldots \ldots \ldots \ldots \\
\text { Light magnesia } \ldots \ldots \ldots \ldots \ldots \ldots \ldots \ldots \ldots \ldots\end{array}$ & $\begin{array}{r}3.2 \mathrm{~kg} \\
2 \mathrm{~kg} \\
2 \mathrm{~kg} \\
28 \mathrm{~kg} \\
450 \mathrm{~g}\end{array}$ & $\begin{array}{l}35 \\
44 \\
44 \\
71 \\
29\end{array}$ & $\begin{array}{l}620 \\
625 \\
626 \\
627 \\
628\end{array}$ & 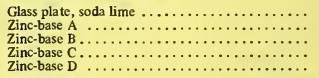 & $\begin{array}{l}\mathrm{pkg}(3) \\
\text { ea } \\
\text { ea } \\
\text { ea } \\
\text { ea }\end{array}$ & $\begin{array}{l}49 \\
54 \\
54 \\
54 \\
54\end{array}$ \\
\hline $\begin{array}{l}377 \\
378 \mathrm{a} \\
379 \\
380 \\
381\end{array}$ & 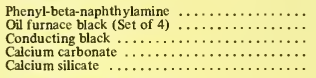 & $\begin{array}{r}600 \mathrm{~g} \\
28 \mathrm{~kg} \\
5.5 \mathrm{~kg} \\
6 \mathrm{~kg} \\
4 \mathrm{~kg}\end{array}$ & $\begin{array}{l}31 \\
40 \\
30 \\
29 \\
29\end{array}$ & $\begin{array}{l}629 \\
630 \\
631 \\
633 \\
634\end{array}$ & 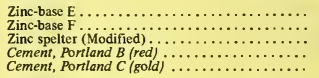 & $\begin{array}{l}\text { ea } \\
\text { ea } \\
\text { ea }\end{array}$ & $\begin{array}{l}54 \\
54 \\
54\end{array}$ \\
\hline $\begin{array}{l}382 \mathrm{a} \\
383 \\
384 \mathrm{a} \\
385 \mathrm{~b}\end{array}$ & $\begin{array}{l}\text { Gas furnace black (Set of } 4) \ldots \ldots \ldots \ldots \ldots \ldots \\
\text { Mercaptobenzothiazole }(\text { Set of } 4) \ldots \ldots \ldots \ldots \ldots \\
\text { N-tertiary-Butyl-2-benzo- } \\
\text { thiazolesulfenamide }(\text { Set of } 4) \ldots \ldots \ldots \ldots \ldots \ldots \ldots \\
\text { Natural rubber } \ldots \ldots \ldots \ldots \ldots \ldots \ldots \ldots \ldots \ldots\end{array}$ & $\begin{array}{r}32 \mathrm{~kg} \\
3.2 \mathrm{~kg} \\
4.5 \mathrm{~kg} \\
31.4 \mathrm{~kg}\end{array}$ & $\begin{array}{r}56 \\
37 \\
63 \\
109\end{array}$ & $\begin{array}{l}635 \\
636 \\
637 \\
638 \\
639\end{array}$ & $\begin{array}{l}\text { Cement, Portland } D \text { (blue) } \ldots \ldots \ldots \ldots \ldots \ldots \ldots \\
\text { Cement, Portland } F \text { (yellow) } \ldots \ldots \ldots \ldots \ldots \ldots \ldots \ldots \\
\text { Cement, Portland } G \text { (pink) } \ldots \ldots \ldots \ldots \ldots \ldots \ldots \ldots \ldots \\
\text { Cement, Portland I (green) } \ldots \ldots \ldots \ldots \ldots \ldots \ldots \ldots \\
\text { Cement, Portland J (clear) } \ldots \ldots \ldots \ldots \ldots \ldots\end{array}$ & & \\
\hline $\begin{array}{l}386 \mathrm{~g} \\
388 \mathrm{f} \\
389 \\
391 \\
404 \mathrm{a}\end{array}$ & $\begin{array}{l}\text { Styrene-butadiene type } 1500 \ldots \ldots \ldots \ldots \ldots \ldots \\
\text { Butyl rubber } \ldots \ldots \ldots \ldots \ldots \ldots \\
\text { Styrene-butadiene, ty pe } 1503 \ldots \ldots \ldots \ldots \ldots \ldots \ldots \\
\text { Acrylonitrile-butadiene rubber } \ldots \ldots \ldots \ldots \ldots \ldots \ldots \ldots \\
\text { Steel, basic electric } \ldots \ldots \ldots \ldots \ldots \ldots \ldots \ldots \ldots\end{array}$ & $\begin{array}{l}34 \mathrm{~kg} \\
37 \mathrm{~kg} \\
34 \mathrm{~kg} \\
25 \mathrm{~kg} \\
\text { ea }\end{array}$ & $\begin{array}{r}71 \\
109 \\
58 \\
109 \\
34\end{array}$ & $\begin{array}{l}641 \\
642 \\
643 \\
644 \\
645\end{array}$ & $\begin{array}{l}\text { Titanium alloy } 8 \mathrm{Mn}(\mathrm{A}) \ldots \ldots \ldots \ldots \ldots \ldots \ldots \\
\text { Titanium alloy } 8 \mathrm{Mn}(\mathrm{B}) \ldots \ldots \ldots \ldots \ldots \ldots \ldots \ldots \ldots \\
\text { Titanium alloy } 8 \mathrm{Mn}(\mathrm{C}) \ldots \ldots \ldots \ldots \ldots \ldots \ldots \\
\text { Titanium alloy } 2 \mathrm{Cr}-2 \mathrm{Fe}-2 \mathrm{Mo}(\mathrm{A}) \ldots \ldots \ldots \ldots \ldots \ldots \ldots \\
\text { Titanium alloy } 2 \mathrm{Cr}-2 \mathrm{Fe}-2 \mathrm{Mo}(\mathrm{B}) \ldots \ldots \ldots \ldots \ldots \ldots\end{array}$ & $\begin{array}{l}\text { ea } \\
\text { ea } \\
\text { ea } \\
\text { ea } \\
\text { ea }\end{array}$ & $\begin{array}{l}54 \\
54 \\
54 \\
54 \\
54\end{array}$ \\
\hline $\begin{array}{l}405 \mathrm{a} \\
407 \mathrm{a} \\
408 \mathrm{a} \\
409 \mathrm{~b} \\
413\end{array}$ & 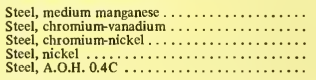 & $\begin{array}{l}\text { ea } \\
\text { ea } \\
\text { ea } \\
\text { ea } \\
\text { ea }\end{array}$ & $\begin{array}{l}34 \\
34 \\
34 \\
34 \\
34\end{array}$ & $\begin{array}{l}646 \\
654 \mathrm{a} \\
661 \\
662 \\
663\end{array}$ & $\begin{array}{l}\text { Titanium alloy } 2 \mathrm{Cr}-2 \mathrm{Fe}-2 \mathrm{Mo}(\mathrm{C}) \ldots \ldots \ldots \ldots \ldots \ldots \\
\text { Titanium alloy, } 6 \mathrm{Al}-4 \mathrm{~V} \\
\text { Steel, AISI } 4340, \text { rod } \ldots \ldots \ldots \ldots \ldots \ldots \ldots \ldots \\
\text { Steel, AlI } 94 \mathrm{~B} 17 \text { (modified), rod } \ldots \ldots \ldots \ldots \ldots \ldots \ldots \\
\text { Steel, Cr-V (modified), rod } \ldots \ldots \ldots \ldots \ldots \ldots \ldots\end{array}$ & $\begin{array}{l}\text { ea } \\
\text { ea } \\
\text { ea } \\
\text { ea } \\
\text { ea }\end{array}$ & $\begin{array}{l}54 \\
39 \\
29 \\
29 \\
29\end{array}$ \\
\hline $\begin{array}{l}414 \\
417 \mathrm{a} \\
418 \\
418 \mathrm{a} \\
420 \mathrm{a}\end{array}$ & 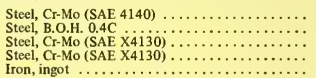 & $\begin{array}{l}\text { ea } \\
\text { ea } \\
\text { ea } \\
\text { ea } \\
\text { ea }\end{array}$ & $\begin{array}{l}34 \\
34 \\
34 \\
34 \\
34\end{array}$ & $\begin{array}{l}664 \\
665 \\
666 \\
667 \\
668\end{array}$ & 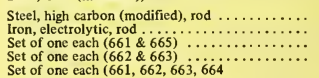 & $\begin{array}{l}\text { ea } \\
\text { ea } \\
\text { set } \\
\text { set }\end{array}$ & $\begin{array}{l}29 \\
29 \\
44 \\
44\end{array}$ \\
\hline $\begin{array}{l}427 \\
431 \\
432 \\
433 \\
434 \\
435\end{array}$ & 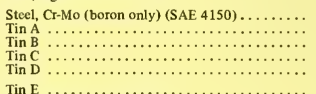 & $\begin{array}{l}\text { ea } \\
\text { ea } \\
\text { ea } \\
\text { ea } \\
\text { ea } \\
\text { ea }\end{array}$ & $\begin{array}{l}34 \\
39 \\
39 \\
39 \\
39 \\
39\end{array}$ & $\begin{array}{l}671 \\
672 \\
673 \\
680 \mathrm{~L}-1 \\
680 \mathrm{~L}-2\end{array}$ & 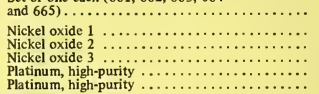 & $\begin{array}{l}\text { set } \\
25 \mathrm{~g} \\
25 \mathrm{~g} \\
25 \mathrm{~g} \\
\mathrm{ea} \\
\mathrm{ea}\end{array}$ & $\begin{array}{r}79 \\
39 \\
39 \\
39 \\
44 \\
194\end{array}$ \\
\hline $\begin{array}{l}435 \\
436 \\
437 \\
438 \\
439 \\
440\end{array}$ & $\begin{array}{l}\text { Tin E } \\
\text { Steel, special Cr6-Mo3-Wio } \ldots \ldots \ldots \ldots \ldots \ldots \\
\text { Steel, special Cr8-Mo 2-W3-Co } 3 \ldots \ldots \ldots \ldots \ldots \\
\text { Steel, Mo high speed (AISI-SAE-Mino) } \\
\text { Steel, Mo high speed (AISI-SAE-M36) } \ldots \ldots \ldots \ldots \\
\text { Steel, special W high speed }\end{array}$ & $\begin{array}{l}\text { ea } \\
\text { ea } \\
\text { ea } \\
\text { ea } \\
\text { ea }\end{array}$ & $\begin{array}{l}39 \\
39 \\
39 \\
39 \\
39\end{array}$ & $\begin{array}{l}680 \mathrm{~L}-2 \\
681 \mathrm{~L}-1 \\
681 \mathrm{~L}-2 \\
682 \\
683 \\
685-\mathrm{R}\end{array}$ & 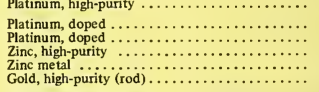 & $\begin{array}{l}\text { ea } \\
\text { ea } \\
\text { ea } \\
\text { ea } \\
\text { ea } \\
\text { ea }\end{array}$ & $\begin{array}{r}194 \\
44 \\
194 \\
94 \\
59 \\
59\end{array}$ \\
\hline $\begin{array}{l}440 \\
441 \\
442 \\
443\end{array}$ & 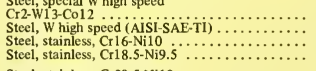 & $\begin{array}{l}\text { ea } \\
\text { ea } \\
\text { ea } \\
\text { ea }\end{array}$ & $\begin{array}{l}39 \\
39 \\
39 \\
39\end{array}$ & $\begin{array}{l}685-W \\
700 c \\
701 c \\
702\end{array}$ & $\begin{array}{l}\text { Gold, high-purity (wire) } \ldots \ldots \ldots \ldots \ldots \ldots \ldots \ldots \\
\text { Paper, light-sensitive } \ldots \ldots \ldots \ldots \ldots \ldots \ldots \ldots \ldots \\
\text { Paper, standard faded strips } \ldots \ldots \ldots \ldots \ldots \ldots \ldots \ldots \ldots \\
\text { Plastic chips, light-sensitive } \ldots \ldots \ldots \ldots \ldots \ldots\end{array}$ & $\begin{array}{r}\text { ea } \\
\text { pkg } \\
\text { bktt } \\
\text { pkg }\end{array}$ & $\begin{array}{r}59 \\
44 \\
159 \\
44 \\
44\end{array}$ \\
\hline $\begin{array}{l}444 \\
445\end{array}$ & $\begin{array}{l}\text { Steel, stainless, Cr20.5-Ni10 } \ldots \\
\text { Steel, stainless, Cr13-Mo0.9 }\end{array}$ & ea & 39 & & Plastic chips, light-sensitive ......... & $\mathrm{pkg}$ & $\begin{array}{l}44 \\
60\end{array}$ \\
\hline 446 & $\begin{array}{l}\text { (Modified AISI } 410 \text { ) } \\
\text { Steel, stainless, Cr18-Nig } \\
\text { (Modified AISI } 321 \text {. } \ldots \ldots \ldots \ldots \ldots \ldots \ldots \ldots \ldots \ldots \ldots \ldots \ldots\end{array}$ & $\begin{array}{l}\text { ea } \\
\text { ea }\end{array}$ & $\begin{array}{l}39 \\
39\end{array}$ & $\begin{array}{l}7049 \\
705 \\
707\end{array}$ & $\begin{array}{l}\text { Polystyrene, narrow molecular weight } \ldots \ldots \ldots \ldots \ldots \\
\text { Polystyrene, broad molecular weight } \ldots \ldots \ldots \ldots \ldots \\
\text { Water vapor permeance, } 12 \text { sheets } \ldots \ldots \ldots \ldots \ldots\end{array}$ & $\begin{array}{l}5 \mathrm{~g} \\
18 \mathrm{~g} \\
\mathrm{pkg}\end{array}$ & $\begin{array}{l}80 \\
87 \\
49\end{array}$ \\
\hline 447 & $\begin{array}{l}\text { Steel, stainless, Cr24-Ni13 } \\
\text { (Modified AISI 309) }\end{array}$ & ea & 39 & 708 & $\begin{array}{l}\text { Glass, relative stress optical } \\
\text { coefficient } \ldots \ldots \ldots \ldots \ldots \ldots \ldots \ldots \ldots \ldots \ldots \ldots \ldots\end{array}$ & & \\
\hline $\begin{array}{l}448 \\
449\end{array}$ & 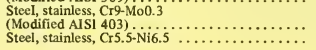 & $\begin{array}{l}\text { ea } \\
\text { ea }\end{array}$ & $\begin{array}{l}39 \\
39\end{array}$ & $\begin{array}{l}709 \\
710\end{array}$ & $\begin{array}{l}\text { Glass, extra dense lead, } \\
4 \times 4 \times 5 \mathrm{~cm} \ldots \ldots \ldots \\
\text { Glass, soda-lime silica... }\end{array}$ & $\begin{array}{l}500 \mathrm{~g} \\
900 \mathrm{~g}\end{array}$ & $\begin{array}{l}75 \\
56\end{array}$ \\
\hline
\end{tabular}




\begin{tabular}{|c|c|c|c|c|c|c|c|}
\hline -SRM & Type & Unit & Price & SRM & Type & Unit & Price \\
\hline $\begin{array}{l}711 \\
712\end{array}$ & $\begin{array}{l}\text { Glass, lead-silica } \ldots \ldots \ldots \ldots \ldots \ldots \ldots \\
\text { Glass, mixed alkali lead silicate } \ldots \ldots \ldots \ldots \ldots \ldots\end{array}$ & $\begin{array}{l}1.3 \mathrm{~kg} \\
225 \mathrm{~g}\end{array}$ & $\begin{array}{r}\$ 79 \\
42\end{array}$ & $\begin{array}{r}837 \\
\text { D837 }\end{array}$ & $\begin{array}{l}\text { Steel, special (Cr8-Mo2-W3-Co3) } \ldots \ldots \ldots \ldots \ldots \ldots \\
\text { Steel, special (Cr8-Mo2-W3-Co3) } \ldots \ldots \ldots \ldots \ldots\end{array}$ & ea & $\$ 47$ \\
\hline $\begin{array}{l}713 \\
714 \\
715\end{array}$ & & $225 \mathrm{~g}$ & $\begin{array}{l}42 \\
42 \\
42\end{array}$ & $\begin{array}{r}838 \\
\mathrm{D} 838 \\
839\end{array}$ & $\begin{array}{l}\text { Steel, Mo high speed (AISI-SAE-M30) } \ldots \ldots \ldots \ldots \\
\text { Steel, Mo high speed (AISI-SAE-M30) } \ldots \ldots \ldots \ldots \\
\text { Steel, Mo high speed (AISI-SAE-M36) } \ldots \ldots \ldots \ldots\end{array}$ & $\begin{array}{l}\text { ea } \\
\text { ea } \\
\text { ea } \\
\text { ea }\end{array}$ & $\begin{array}{l}47 \\
54 \\
47\end{array}$ \\
\hline $\begin{array}{l}715 \\
716 \\
717\end{array}$ & $\begin{array}{l}\text { Glass, alkali-free aluminosilicate } \ldots \ldots \ldots \ldots \ldots \ldots \\
\text { Glass, neutral (borosilicate) } \ldots \ldots \ldots \ldots \ldots \ldots \ldots \\
\text { Glass, standard, borosilicate } \ldots \ldots \ldots \ldots \ldots\end{array}$ & $\begin{array}{l}200 \mathrm{~g} \\
250 \mathrm{~g} \\
450 \mathrm{~g}\end{array}$ & $\begin{array}{l}42 \\
42 \\
75\end{array}$ & $\begin{array}{r}\mathrm{D} 839 \\
840\end{array}$ & $\begin{array}{l}\text { Steel, Mo high speed (AISI-SAE-M36) } \ldots \ldots \ldots \\
\text { Steel, special W high speed }\end{array}$ & ea & 54 \\
\hline $\begin{array}{l}718 \\
720 \\
723\end{array}$ & $\begin{array}{l}\text { Polycrystalline alumina, Elasticity } \ldots \ldots \ldots \ldots \ldots \\
\text { Sapphire, synthetic }\left(\mathrm{AI}_{2} \mathrm{O}_{3}\right) \ldots \ldots \ldots \ldots \ldots \ldots \\
\text { Tris(hydroxy methyl)aminomethane, }\end{array}$ & $15 \mathrm{~g}$ & $\begin{array}{r}199 \\
60\end{array}$ & D840 & 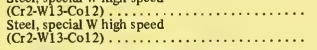 & $\begin{array}{l}\text { ea } \\
\text { ea }\end{array}$ & $\begin{array}{l}47 \\
54\end{array}$ \\
\hline & 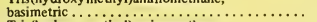 & $50 \mathrm{~g}$ & 55 & 841 & Steel, W high speed (AISI-SAE-T1) . . . . . . . . & ea & 47 \\
\hline 724 & $\begin{array}{l}\text { Tris(hydroxymethyl)aminomethane, } \\
\text { calorimetric } \ldots \ldots \ldots \ldots \ldots \ldots \ldots \ldots \ldots \ldots\end{array}$ & $50 \mathrm{~g}$ & 44 & D841 & $\begin{array}{l}\text { Steel, W high speed (AISI-SAE-T1) } \ldots \ldots \ldots \ldots \ldots \\
\text { Steel, Cr13-Mo0.9 (Modified AISI } 410) \ldots \ldots \ldots\end{array}$ & ea & $\begin{array}{l}54 \\
47\end{array}$ \\
\hline $\begin{array}{l}725 \\
726\end{array}$ & $\begin{array}{l}\text { Mossbauer Differential Chemical Shift } \ldots \ldots \ldots \ldots \\
\text { Selenium }\end{array}$ & $450 \mathrm{~g}$ & $\begin{array}{r}159 \\
49\end{array}$ & $\begin{array}{l}\text { D845 } \\
\text { D846 }\end{array}$ & $\begin{array}{l}\text { Steel, Cr13-Mo0.9 (Modified AIS1 } 410) \ldots \ldots \ldots \\
\text { Steel, Cr18-Ni9 (Modified AIS1 321) } \ldots \ldots \ldots \ldots\end{array}$ & ea & $\begin{array}{l}54 \\
54\end{array}$ \\
\hline $\begin{array}{l}728 \\
731 \mathrm{~L} 1\end{array}$ & $\begin{array}{l}\text { Zinc } \\
\text { Borosilicate glass, thermal } \\
\text { expansion, } 2 \text { in. } \ldots \ldots \ldots \ldots \ldots \ldots \ldots \ldots \ldots \ldots \ldots\end{array}$ & $450 \mathrm{~g}$ & $\begin{array}{l}47 \\
47\end{array}$ & $\begin{array}{r}\text { D847 } \\
849 \\
\text { D849 }\end{array}$ & 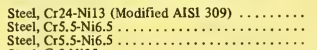 & $\begin{array}{l}\text { ea } \\
\text { ea } \\
\text { ea }\end{array}$ & $\begin{array}{l}54 \\
47 \\
54\end{array}$ \\
\hline $731 \mathrm{~L} 2$ & $\begin{array}{l}\text { Borosilicate glass, thermal } \\
\text { expansion, } 4 \text { in. } \ldots \ldots \ldots \ldots \ldots \ldots \ldots \ldots \ldots \ldots\end{array}$ & ea & 123 & $\begin{array}{r}1849 \\
850 \\
\text { D850 }\end{array}$ & 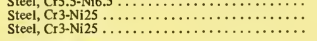 & ea & $\begin{array}{l}54 \\
47 \\
54\end{array}$ \\
\hline $731 \mathrm{~L} 3$ & Borosilicate glass, thermal & & & 911 & Cholesterol, clinical $\ldots \ldots \ldots \ldots \ldots \ldots \ldots$ & $0.5 \mathrm{~g}$ & 34 \\
\hline 733 & 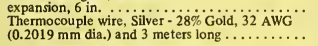 & ea & $\begin{array}{r}171 \\
89\end{array}$ & $\begin{array}{l}912 \\
913 \\
914\end{array}$ & $\begin{array}{l}\text { Urea, clinical } \\
\text { Uric acid, clinical } \ldots \ldots \ldots \ldots \ldots \ldots \ldots \ldots \ldots \ldots \ldots \ldots \ldots \\
\text { Creatinine, clinical } \ldots \ldots \ldots \ldots \ldots \ldots\end{array}$ & $\begin{array}{l}25 \mathrm{~g} \\
10 \mathrm{~g} \\
10 \mathrm{~g}\end{array}$ & $\begin{array}{l}40 \\
34 \\
40\end{array}$ \\
\hline $734 \mathrm{~S}$ & Iron, electroly tic, thermal conductivity, & & & 915 & 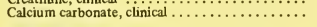 & $20 \mathrm{~g}$ & 34 \\
\hline $734 \mathrm{~L} 1$ & $\begin{array}{l}\text { rod } 6.4 \mathrm{~mm} \text { dia, }, 305 \mathrm{~mm} \text { long } \ldots \ldots \ldots \ldots \ldots \\
\text { Iron, electrolytic, thermal conductivity, }\end{array}$ & ea & 79 & $\begin{array}{l}916 \\
917\end{array}$ & $\begin{array}{l}\text { Bilirubin, clinical } \ldots \ldots \ldots \ldots \ldots \ldots \ldots \ldots \ldots \ldots \ldots \ldots \\
\text { D-Glucose, clinical } \ldots \ldots \ldots \ldots \ldots \ldots \ldots\end{array}$ & $\begin{array}{c}100 \mathrm{mg} \\
25 \mathrm{~g}\end{array}$ & $\begin{array}{l}96 \\
47\end{array}$ \\
\hline $734 \mathrm{~L} 2$ & $\begin{array}{l}\text { rod, } 31.8 \mathrm{~mm} \text { dia., } 152 \mathrm{~mm} \text { long } \ldots \ldots \ldots \ldots \ldots \\
\text { lron, electroly tic, thermal conductivity, } \\
\text { rod } 31.8 \mathrm{~mm} \text { dia., } 305 \mathrm{~mm} \text { long } \ldots \ldots \ldots \ldots \ldots\end{array}$ & $\begin{array}{l}\text { ea } \\
\text { ea }\end{array}$ & $\begin{array}{r}89 \\
154\end{array}$ & $\begin{array}{l}911 \\
919 \\
920\end{array}$ & 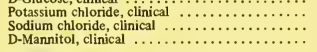 & $\begin{array}{l}30 \mathrm{~g} \\
30 \mathrm{~g} \\
50 \mathrm{~g}\end{array}$ & $\begin{array}{l}44 \\
44 \\
61\end{array}$ \\
\hline $735 S$ & $\begin{array}{l}\text { Stainless steel, thermal conductivity, } \\
\text { rod } 0.65 \mathrm{~cm} \text { dia., } 30 \mathrm{~cm} \text { long } \ldots \ldots \ldots \ldots \ldots\end{array}$ & & & $\begin{array}{l}921 \\
922\end{array}$ & $\begin{array}{l}\text { Cortisol ........................................ } \\
\text { Tris(hydroxy methyl)aminomethane }\end{array}$ & & \\
\hline $735 \mathrm{M} 1$ & $\begin{array}{l}\text { Stainless steel, thermal conductivity, } \\
\text { rod } 1.25 \mathrm{~cm} \text { dia., } 15 \mathrm{~cm} \text { long } \ldots \ldots \ldots \ldots \ldots \ldots\end{array}$ & ea & 104 & 923 & & $25 \mathrm{~g}$ & 44 \\
\hline $735 \mathrm{M} 2$ & $\begin{array}{l}\text { Stainiess steel, thermal conductivity, } \\
\text { rod } 1.25 \mathrm{~cm} \text { dia., } 30 \mathrm{~cm} \text { long } \ldots \ldots \ldots \ldots \ldots .\end{array}$ & ea & 154 & 320 & hydrochloride, clinical $\ldots \ldots \ldots \ldots \ldots \ldots \ldots$ & $35 \mathrm{~g}$ & 44 \\
\hline $735 L 1$ & $\begin{array}{l}\text { Stainless steel, thermal conductivity, } \\
\text { rod } 3.5 \mathrm{~cm} \text { dia., } 5 \mathrm{~cm} \text { long } \ldots \ldots \ldots \ldots \ldots \ldots\end{array}$ & & & $\begin{array}{l}924 \\
925\end{array}$ & 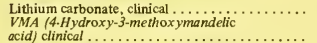 & $30 \mathrm{~g}$ & 54 \\
\hline $735 L 2$ & $\begin{array}{l}\text { Stainless steel, thermal conductivity, } \\
\text { rod } 3.5 \mathrm{~cm} \text { dia., } 10 \mathrm{~cm} \text { long } \\
\text { Copper, thermal expansion, } 2 \text { in. . . . . . . . . }\end{array}$ & & & $930 \mathrm{a}$ & 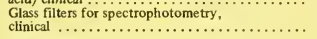 & $\operatorname{set}(3)$ & 304 \\
\hline $\begin{array}{l}736 \mathrm{~L} 1 \\
736 \mathrm{~L} 2\end{array}$ & $\begin{array}{l}\text { Copper, thermal expansion, } 2 \text { in. } \ldots \ldots \ldots \ldots \ldots \\
\text { Copper, thermal expansion, } 4 \text { in. } \ldots \ldots \ldots \ldots \ldots\end{array}$ & $\begin{array}{l}\text { ea } \\
\text { ea }\end{array}$ & $\begin{array}{r}75 \\
123\end{array}$ & 931 & Liquid filters for spectrophotometry, & & \\
\hline $\begin{array}{l}736 \mathrm{LL} \\
736 \mathrm{~L} 3 \\
737 \mathrm{~L} 1 \\
737 \mathrm{L2} \\
737 \mathrm{L3}\end{array}$ & 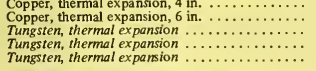 & $\begin{array}{l}\text { ea } \\
\text { ea }\end{array}$ & $\begin{array}{l}123 \\
171\end{array}$ & $\begin{array}{l}944 \\
945 \\
946\end{array}$ & $\begin{array}{l}\text { clinical, } 3 \text { sets of } 4 \text {. } \ldots \ldots \ldots \ldots \ldots \\
\text { Plutonium sulfate tetrahydrate assay } \ldots \ldots \ldots \ldots \ldots \\
\text { Plutonium metal, std matrix } \ldots \ldots \ldots \ldots \ldots \ldots \ldots \\
\text { Plutonium, } 12 \% \text { isotopic } \ldots \ldots \ldots \ldots \ldots\end{array}$ & $\begin{array}{r}\text { set } \\
0.5 \mathrm{~g} \\
5 \mathrm{~g} \\
0.25 \mathrm{~g}\end{array}$ & $\begin{array}{r}69 \\
80 \\
504 \\
154\end{array}$ \\
\hline $\begin{array}{l}739 \mathrm{~L} 1 \\
739 \mathrm{~L} 2 \\
739 \mathrm{~L} 3 \\
740 \\
741\end{array}$ & $\begin{array}{l}\text { Fused-silica, thermal expansion, } 2 \text { in. } \ldots \ldots \ldots \ldots \\
\text { Fused-silica, thermal expansion, } 4 \text { in. } \ldots \ldots \ldots \ldots \\
\text { Fused-silica, thermal expansion, } 6 \text { in. } \\
\text { Zinc, primary freezing-point std. } \ldots \ldots \ldots \ldots \ldots \\
\text { Tin, primary freezing-point std. } \ldots \ldots \ldots \ldots\end{array}$ & $\begin{array}{l}\text { ea } \\
\text { ea } \\
\text { ea } \\
350 \mathrm{~g} \\
350 \mathrm{~g}\end{array}$ & $\begin{array}{r}75 \\
123 \\
171 \\
100 \\
125\end{array}$ & $\begin{array}{l}947 \\
948 \\
949 \mathrm{~d} \\
950 \mathrm{a} \\
951\end{array}$ & 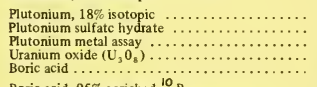 & $\begin{array}{r}0.25 \mathrm{~g} \\
0.25 \mathrm{~g} \\
0.5 \mathrm{~g} \\
25 \mathrm{~g} \\
100 \mathrm{~g}\end{array}$ & $\begin{array}{r}154 \\
71 \\
154 \\
32 \\
59\end{array}$ \\
\hline $\begin{array}{l}742 \\
745 \\
746 \\
747 \\
748\end{array}$ & $\begin{array}{l}\text { Alumina, high temperature melting point } \ldots \ldots \ldots \\
\text { Gold, vapor pressure std. } \ldots \ldots \ldots \ldots \ldots \ldots \ldots \ldots \\
\text { Cadmium, vapor pressure std. } \ldots \ldots \ldots \ldots \ldots \ldots \ldots \\
\text { Platinum, vapor pressure std. } \ldots \ldots \ldots \ldots \ldots \ldots \\
\text { Silver, vapor pressure std. } \ldots \ldots \ldots \ldots \ldots\end{array}$ & $\begin{array}{l}10 \mathrm{~g} \\
\text { ea }\end{array}$ & $\begin{array}{l}67 \\
89 \\
69 \\
79\end{array}$ & $\begin{array}{l}952 \\
953 \\
953-\mathrm{L} 1 \\
953-\mathrm{L} 2 \\
953 \cdot \mathrm{L} 3\end{array}$ & $\begin{array}{l}\text { Boric acid, } 95 \% \text { enriched }{ }^{10} \mathrm{~B}, \ldots \ldots \ldots \\
\text { Neutron density monitor wire, } 1 \text { meter long } \ldots \ldots \\
\text { Neutron density monitor wire, } 5 \text { meters long ..... } \\
\text { Neutron density monitor wirc, } 10 \text { metcrs long } \ldots . \\
\text { Neutron density monitor wire, } 25 \text { meters long } \ldots\end{array}$ & $\begin{array}{l}0.25 \mathrm{~g} \\
\mathrm{ea} \\
\mathrm{ea} \\
\mathrm{ea} \\
\mathrm{ea}\end{array}$ & $\begin{array}{r}44 \\
43 \\
100 \\
171 \\
385\end{array}$ \\
\hline $\begin{array}{l}740 \\
755 \\
756 \\
758 \\
759\end{array}$ & 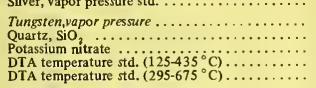 & $\begin{array}{l}2 \mathrm{~g} \\
5 \mathrm{~g} \\
\operatorname{set}(5) \\
\operatorname{set}(5)\end{array}$ & $\begin{array}{l}39 \\
39 \\
49 \\
49\end{array}$ & $\begin{array}{l}960 \\
975 \\
976 \\
977 \\
978\end{array}$ & 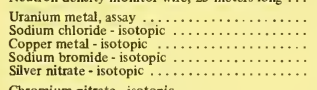 & $\begin{array}{l}26 \mathrm{~g} \\
0.25 \mathrm{~g} \\
0.25 \mathrm{~g} \\
0.25 \mathrm{~g} \\
0.25 \mathrm{~g}\end{array}$ & $\begin{array}{r}503 \\
54 \\
44 \\
44 \\
44\end{array}$ \\
\hline $\begin{array}{l}760 \\
763-1 \\
763-2\end{array}$ & $\begin{array}{l}\text { DTA temperature std. }\left(570-940^{\circ} \mathrm{C}\right) \ldots \ldots \ldots \ldots \\
\text { Aluminum, magnetic susceptibility, } \\
\text { cylinder } \ldots \ldots \ldots \ldots \ldots \ldots \ldots \ldots \ldots \ldots \\
\text { Aluminum, magnetic susceptibility, } \\
\text { wire } \ldots \ldots \ldots \ldots \ldots \ldots \ldots \ldots \ldots \ldots \ldots \ldots \ldots\end{array}$ & $\begin{array}{l}\operatorname{set}(3) \\
\operatorname{set}(5)\end{array}$ & $\begin{array}{l}49 \\
49\end{array}$ & $\begin{array}{l}979 \\
980 \\
981-3 \\
984 \\
987\end{array}$ & 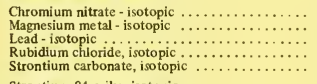 & $\begin{array}{r}0.25 \mathrm{~g} \\
0.25 \mathrm{~g} \\
\text { set } \\
1 \mathrm{~g} \\
1 \mathrm{~g}\end{array}$ & $\begin{array}{r}44 \\
44 \\
109 \\
47 \\
44\end{array}$ \\
\hline $\begin{array}{l}763-3 \\
7641 \\
7642\end{array}$ & 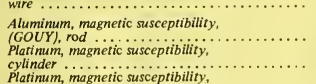 & & & $\begin{array}{c}988 \\
999 \\
1000 \\
1002 \mathrm{~b} \\
1003\end{array}$ & 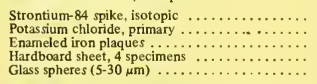 & $\begin{array}{l}1 \mathrm{mg} \\
60 \mathrm{~g} \\
\text { set( }(3) \\
\text { sct } \\
40 \mathrm{~g}\end{array}$ & $\begin{array}{r}154 \\
57 \\
29 \\
39 \\
37\end{array}$ \\
\hline 7642 & $\begin{array}{l}\text { Platinum, magnetic susceptibility, } \\
\text { wire } \ldots \ldots \ldots \ldots \ldots \ldots \ldots \ldots \ldots \ldots \ldots \ldots \ldots \ldots \ldots\end{array}$ & & & $\begin{array}{l}1004 \\
1006\end{array}$ & $\begin{array}{l}\text { Glass beads } \ldots \ldots \ldots \ldots \ldots \ldots \ldots \ldots \ldots \ldots \\
\text { Smoke density std., non-flaming } \ldots \ldots \ldots \ldots \ldots\end{array}$ & $\begin{array}{c}63 \mathrm{~g} \\
\mathrm{pkg}(3)\end{array}$ & 52 \\
\hline $\begin{array}{l}765 \cdot 1 \\
765-2\end{array}$ & $\begin{array}{l}\text { Palladium, magnetic susceptibility, } \\
\text { cylinder .............................. } \\
\text { Palladium, magnetic susceptibility, }\end{array}$ & & & $\begin{array}{l}1007 \\
1008 \\
1009\end{array}$ & $\begin{array}{l}\text { Smoke density std., flaming } \ldots \ldots \ldots \ldots \ldots \ldots \ldots \\
\text { Photographic step tablet, } 0-4 \ldots \ldots \ldots \ldots \ldots \\
\text { Photographic step tablet } 0-3 \ldots \ldots \ldots \ldots \ldots\end{array}$ & $\begin{array}{l}\mathrm{pkg}(3) \\
\text { ea } \\
\text { ea }\end{array}$ & $\begin{array}{l}34 \\
34 \\
72 \\
58\end{array}$ \\
\hline $765-3$ & 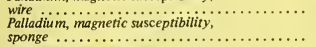 & & & $\begin{array}{l}1010 \mathrm{a} \\
1011 \\
1013\end{array}$ & $\begin{array}{l}\text { Microcopy test chart } \ldots \ldots \ldots \ldots \ldots \ldots \ldots \ldots \ldots \\
\text { Cement, Portland } \ldots \ldots \ldots \ldots \ldots \ldots\end{array}$ & set & 14 \\
\hline $766 \cdot 1$ & $\begin{array}{l}\text { Manganese Fluoride, magnetic } \\
\text { susceptibility, cube } . \ldots \ldots \ldots \ldots \ldots \ldots \ldots \ldots\end{array}$ & & & $\begin{array}{l}1013 \\
1014 \\
1015\end{array}$ & $\begin{array}{l}\text { Cement, Portland } \ldots \ldots \ldots \ldots \ldots \ldots \ldots \ldots \ldots \ldots \ldots \ldots \ldots \ldots \\
\text { Cement, Portland } \ldots \ldots \ldots \ldots \ldots \ldots \ldots \ldots \ldots \ldots \\
\text { Cement, Portland } \ldots \ldots \ldots \ldots \ldots \ldots \ldots\end{array}$ & $\begin{array}{l}\text { set } \\
\text { set } \\
\text { set }\end{array}$ & $\begin{array}{l}32 \\
32 \\
32\end{array}$ \\
\hline $\begin{array}{r}767 \\
803 a \\
\text { D803a }\end{array}$ & 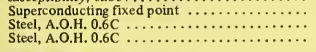 & $\begin{array}{l}\text { ea } \\
\text { ea } \\
\text { ea }\end{array}$ & $\begin{array}{r}250 \\
34 \\
39\end{array}$ & $\begin{array}{l}1016 \\
1017 \mathrm{a} \\
1018 \mathrm{a}\end{array}$ & $\begin{array}{l}\text { Cement, Portland } \ldots \ldots \ldots \ldots \ldots \ldots \\
\left.\text { Glass beads (sieve nos. } \text { so }^{2} 140\right) \ldots \ldots \ldots \ldots \ldots \ldots \\
\text { Glass beads (sieve nos. } 25-60) \ldots \ldots \ldots \ldots \ldots\end{array}$ & $\begin{array}{l}\text { set } \\
84 \mathrm{~g} \\
74 \mathrm{~g}\end{array}$ & $\begin{array}{l}32 \\
44 \\
44\end{array}$ \\
\hline $\begin{array}{r}804 a \\
805 a\end{array}$ & Steel, basic electric . . . . . . . . . . . . . . . . . . . . . . . & ea & $\begin{array}{l}34 \\
34 \\
39\end{array}$ & $\begin{array}{l}1018 \mathrm{a} \\
1019 \\
1020\end{array}$ & $\begin{array}{l}\text { Glass beads (sleve nos. } 25-60) \ldots \ldots \ldots \ldots \ldots \ldots \\
\text { Glass spheres (sieves No.8-18) } \ldots \ldots \ldots \ldots \ldots \ldots \ldots \\
\text { Zinc sulfide phosphor } \ldots \ldots \ldots \ldots \ldots \ldots \ldots\end{array}$ & $\begin{array}{r}74 \mathrm{~g} \\
100 \mathrm{~g} \\
14 \mathrm{~g}\end{array}$ & $\begin{array}{l}44 \\
35 \\
28\end{array}$ \\
\hline D805a & Steel, medium manganese $\ldots \ldots \ldots \ldots \ldots \ldots \ldots$ & ea & $\begin{array}{l}39 \\
34\end{array}$ & 1021 & Zinc silicate phosphor $\ldots \ldots \ldots \ldots \ldots \ldots \ldots \ldots$ & $28 \mathrm{~g}$ & 28 \\
\hline $\begin{array}{r}807 \mathrm{a} \\
\mathrm{D} 807 \mathrm{a}\end{array}$ & $\begin{array}{l}\text { Steel, chromium-vanadium } \ldots \ldots \ldots \ldots \ldots \ldots \ldots \ldots \\
\text { Steel, chromium-vanadium .................. }\end{array}$ & ea & $\begin{array}{l}34 \\
39\end{array}$ & $\begin{array}{l}1022 \\
1023\end{array}$ & $\begin{array}{l}\text { Zinc sulfide phosphor } \\
\text { Zinc-cadmium sulfide phosphor }\end{array}$ & $14 \mathrm{~g}$ & 28 \\
\hline $\begin{array}{l}808 \mathrm{a} \\
809 \mathrm{~b}\end{array}$ & $\begin{array}{l}\text { Steel, chromium-nickel } \ldots \ldots \ldots \ldots \ldots \ldots \ldots \ldots \ldots \\
\text { Steel, nickel } \ldots \ldots \ldots \ldots \ldots \ldots \ldots \ldots \ldots\end{array}$ & $\begin{array}{l}\text { ea } \\
\text { ea }\end{array}$ & $\begin{array}{l}34 \\
34\end{array}$ & 1024 & $\begin{array}{l}\text { Linc-cadivium sulmae phosphor } \\
\text { Zinc-cadmium sulfide phosphor }\end{array}$ & $14 \mathrm{~g}$ & 30 \\
\hline D809b & 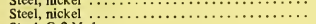 & ea & 39 & 1024 & 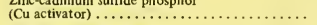 & $14 \mathrm{~g}$ & 28 \\
\hline $\begin{array}{l}810 \mathrm{a} \\
817 \mathrm{a}\end{array}$ & 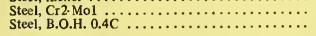 & ea & $\begin{array}{l}34 \\
34\end{array}$ & $\begin{array}{l}1025 \\
1026\end{array}$ & $\begin{array}{l}\text { Zinc phosphate phosphor } \ldots \ldots \ldots \ldots \ldots \ldots \ldots \\
\text { Calcium tungstate phosphor } \ldots \ldots \ldots \ldots \ldots \ldots\end{array}$ & $28 \mathrm{~g}$ & $\begin{array}{l}28 \\
28\end{array}$ \\
\hline $\begin{array}{r}820 \mathrm{a} \\
\text { D820a } \\
821 \\
827\end{array}$ & 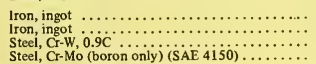 & $\begin{array}{l}\text { ea } \\
\text { ea } \\
\text { ea } \\
\text { ea }\end{array}$ & $\begin{array}{l}34 \\
39 \\
34 \\
34\end{array}$ & $\begin{array}{l}1026 \\
1027 \\
1028 \\
1029\end{array}$ & $\begin{array}{l}\text { Magnesium tungstate phosphor } \ldots \ldots \ldots \ldots \ldots \ldots \ldots \ldots \\
\text { Zinc silicate phosphor } \ldots \ldots \ldots \ldots \ldots \ldots \ldots \ldots \\
\text { Calcium silicate phosphor } \ldots \ldots \ldots \ldots \ldots \ldots\end{array}$ & $\begin{array}{l}28 \mathrm{~g} \\
28 \mathrm{~g} \\
28 \mathrm{~g} \\
14 \mathrm{~g}\end{array}$ & $\begin{array}{l}28 \\
28 \\
28 \\
28\end{array}$ \\
\hline
\end{tabular}




\begin{tabular}{|c|c|c|c|c|c|c|c|}
\hline SRM & Type & Unit & Price & SRM & Type & Unit & Price \\
\hline $\begin{array}{l}1030 \\
1031 \\
1032 \\
1033 \\
1051 \mathrm{~b}\end{array}$ & 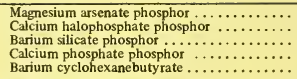 & $\begin{array}{r}28 \mathrm{~g} \\
28 \mathrm{~g} \\
28 \mathrm{~g} \\
28 \mathrm{~g} \\
5 \mathrm{~g}\end{array}$ & $\begin{array}{l}\$ 28 \\
28 \\
28 \\
28 \\
35\end{array}$ & $\begin{array}{l}1132 \\
1134 \\
1135 \\
1136 \\
1138\end{array}$ & 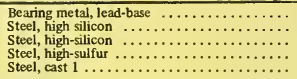 & $\begin{array}{l}\text { ea } \\
\text { ea } \\
\text { ea } \\
\text { ea } \\
\text { ea }\end{array}$ & $\begin{array}{r}\$ 54 \\
54 \\
54 \\
54 \\
69\end{array}$ \\
\hline $\begin{array}{l}1052 b \\
1053 a \\
1055 b \\
1057 b\end{array}$ & $\begin{array}{l}\text { Bis(1-phenyl-1,3-butanediono) } \\
\text { oxovanadium (IV) } \ldots \ldots \ldots \ldots \ldots \ldots \ldots \ldots \\
\text { Cadmium cyclohexanebuty rate } \ldots \ldots \ldots \ldots \ldots \ldots \\
\text { Cobalt cyclohexanebutyrate } \ldots \ldots \ldots \ldots \ldots \\
\text { Dibutyltin bis(2-ethylhexanoate) } \ldots \ldots \ldots \ldots \ldots\end{array}$ & $\begin{array}{l}5 \mathrm{~g} \\
5 \mathrm{~g} \\
5 \mathrm{~g} \\
5 \mathrm{~g}\end{array}$ & $\begin{array}{l}35 \\
35 \\
35 \\
35\end{array}$ & $\begin{array}{l}1139 \\
1140 \\
1141 \\
1142 \\
1143\end{array}$ & 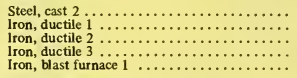 & $\begin{array}{l}\text { ea } \\
\text { ea } \\
\text { ea } \\
\text { ea } \\
\text { ea }\end{array}$ & $\begin{array}{l}69 \\
69 \\
69 \\
69 \\
69\end{array}$ \\
\hline $\begin{array}{l}1059 \mathrm{~b} \\
1060 \mathrm{a} \\
1061 \mathrm{c} \\
1062 \mathrm{a} \\
1063 \mathrm{a}\end{array}$ & $\begin{array}{l}\text { Lead cyclohexanebutyrate } \ldots \ldots \ldots \ldots \ldots \ldots \\
\text { Lithium cyclohexanebuty yate } \ldots \ldots \ldots \ldots \ldots \ldots \\
\text { Magnesium cyclohexanebutyrate } \ldots \ldots \ldots \ldots \ldots \ldots \\
\text { Manganous cyclohexanebutyrate } \ldots \ldots \ldots \ldots \ldots \\
\text { Methyl borate } \ldots \ldots \ldots \ldots \ldots \ldots \ldots \ldots \ldots \ldots\end{array}$ & $\begin{array}{l}5 \mathrm{~g} \\
5 \mathrm{~g} \\
5 \mathrm{~g} \\
5 \mathrm{~g} \\
5 \mathrm{~g}\end{array}$ & $\begin{array}{l}35 \\
35 \\
35 \\
35 \\
35\end{array}$ & $\begin{array}{l}1144 \\
1147 \\
1148 \\
1149 \\
1152\end{array}$ & 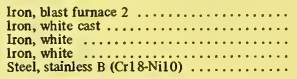 & $\begin{array}{l}\text { ea } \\
\text { ea } \\
\text { ea } \\
\text { ea } \\
\text { ea }\end{array}$ & $\begin{array}{l}69 \\
69 \\
69 \\
69 \\
69\end{array}$ \\
\hline $\begin{array}{l}1064 \\
1065 \mathrm{~b} \\
1066 \mathrm{a} \\
1069 \mathrm{~b} \\
1070 \mathrm{a}\end{array}$ & 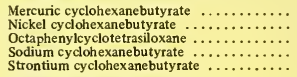 & $\begin{array}{l}5 \mathrm{~g} \\
5 \mathrm{~g} \\
5 \mathrm{~g} \\
5 \mathrm{~g} \\
5 \mathrm{~g}\end{array}$ & $\begin{array}{l}35 \\
35 \\
35 \\
35 \\
35\end{array}$ & $\begin{array}{l}1154 \\
1155 \\
1156 \\
1157 \\
1158\end{array}$ & $\begin{array}{l}\text { Steel, stainless D (Cr19-Ni10) } \ldots \ldots \ldots \ldots \ldots \\
\text { Steel, stainless, Cr18-Ni12-Mor } 2 \ldots \ldots \ldots \ldots \ldots \ldots \\
\text { Steel, maraging (disk form) } \ldots \ldots \ldots \ldots \ldots \ldots \ldots \\
\text { Steel, tool } \\
\text { Steel, high nickel ( } 36 \% \text { Ni) } \ldots \ldots \ldots \ldots \ldots \ldots \ldots\end{array}$ & $\begin{array}{l}\text { ea } \\
\text { ea } \\
\text { ea } \\
\text { ea } \\
\text { ea }\end{array}$ & $\begin{array}{l}69 \\
69 \\
69 \\
54 \\
54\end{array}$ \\
\hline $\begin{array}{l}1071 \mathrm{a} \\
1073 \mathrm{~b} \\
1074 \mathrm{a} \\
1075 \mathrm{a} \\
1076\end{array}$ & $\begin{array}{l}\text { Triphenyl phosphate } \ldots \ldots \ldots \ldots \ldots \ldots \ldots \ldots \\
\text { Zinc cyclohexanebutyrate } \ldots \ldots \ldots \ldots \ldots \ldots \ldots \ldots \\
\text { Calcium 2-ethylhexanoate } \ldots \ldots \ldots \ldots \ldots \ldots \ldots \ldots \\
\text { Aluminum 2-ethylhexanoate } \\
\text { Potassium erucate } \ldots \ldots \ldots \ldots \ldots \ldots \ldots \ldots \ldots \ldots\end{array}$ & $\begin{array}{l}5 \mathrm{~g} \\
5 \mathrm{~g} \\
5 \mathrm{~g} \\
5 \mathrm{~g} \\
5 \mathrm{~g}\end{array}$ & $\begin{array}{l}35 \\
35 \\
35 \\
35 \\
35\end{array}$ & $\begin{array}{l}1159 \\
1160 \\
1165 \\
1166 \\
1167\end{array}$ & 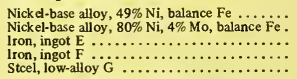 & $\begin{array}{l}\text { ea } \\
\text { ea } \\
\text { ea } \\
\text { ea } \\
\text { ea }\end{array}$ & $\begin{array}{l}69 \\
69 \\
69 \\
69 \\
69\end{array}$ \\
\hline $\begin{array}{l}1077 \mathrm{a} \\
1078 \mathrm{~b} \\
1079 \mathrm{~b}\end{array}$ & 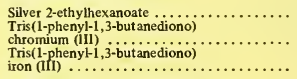 & $\begin{array}{l}5 \mathrm{~g} \\
5 \mathrm{~g} \\
5 \mathrm{~g}\end{array}$ & $\begin{array}{l}35 \\
37 \\
35\end{array}$ & $\begin{array}{l}1171 \\
1172 \\
1185 \\
1197 \\
1198\end{array}$ & 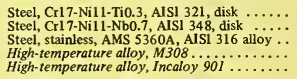 & $\begin{array}{l}\text { ea } \\
\text { ea } \\
\text { ea }\end{array}$ & $\begin{array}{l}54 \\
54 \\
69\end{array}$ \\
\hline $\begin{array}{l}1080 \\
1089 \\
1090\end{array}$ & 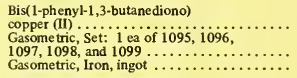 & $\begin{array}{c}5 g \\
\text { set(5) } \\
\text { ea }\end{array}$ & $\begin{array}{l}35 \\
79 \\
59\end{array}$ & $\begin{array}{l}1199 \\
1200 \\
1201 \\
1206-2 \\
1207-1\end{array}$ & $\begin{array}{l}\text { High-temperature alloy, } L 605 \ldots \ldots \ldots \ldots \ldots \\
\text { High-emperature alloy, S816 } \ldots \ldots \ldots \ldots \ldots \ldots \\
\text { High-temperature alloy, Hastaloy } ¥ \ldots \ldots \ldots \ldots \\
\text { High temperature alloy, Rene } 41 \ldots \ldots \ldots \ldots \\
\text { High temperature alloy, Waspaloy (No. i) } \ldots\end{array}$ & $\begin{array}{l}\text { ea } \\
\text { ea }\end{array}$ & $\begin{array}{l}54 \\
54\end{array}$ \\
\hline $\begin{array}{l}1091 \\
1092 \\
1093 \\
1094\end{array}$ & 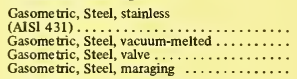 & $\begin{array}{l}\text { ea } \\
\text { ea } \\
\text { ea } \\
\text { ea }\end{array}$ & $\begin{array}{l}59 \\
59 \\
59 \\
59\end{array}$ & $\begin{array}{l}1207-2 \\
1208-1 \\
1208-2 \\
1209\end{array}$ & $\begin{array}{l}\text { High temperature alloy, Waspaloy (No. 2) } \ldots . \\
\text { High temperature alloy, nco } 718 \text { (No. } 1) \ldots \text {. } \\
\text { High temperature alloy, Inco } 718 \text { (No. } 2) \ldots \ldots \\
\text { High temperature alloy, Set, } 1 \text { ea of } 1206-2, \\
1207-1,1207-2,1208-1 \text {, and } 1208-2 \ldots \ldots\end{array}$ & $\begin{array}{l}\text { ea } \\
\text { ea } \\
\text { ea } \\
\text { set }\end{array}$ & $\begin{array}{r}54 \\
54 \\
54 \\
189\end{array}$ \\
\hline $\begin{array}{l}1095 \\
1096 \\
1097\end{array}$ & $\begin{array}{l}\text { Gasometric, Steel, AISI 4340, } \\
\text { rod ........... } \\
\text { Gasometric, Steel, Aisi } 94 \mathrm{Bi} 7 \\
\text { (modified), } \\
\text { Gasometric, Steel, Cir-v (modified), }\end{array}$ & ea & 37 & $\begin{array}{l}1210 \\
1261 \\
1262 \\
1263 \\
1264\end{array}$ & 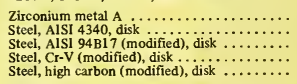 & $\begin{array}{l}\text { ea } \\
\text { ea } \\
\text { ea } \\
\text { ea } \\
\text { ea }\end{array}$ & $\begin{array}{l}94 \\
49 \\
49 \\
49 \\
49\end{array}$ \\
\hline 1098 & $\begin{array}{l}\text { gad } \ldots \ldots \ldots \ldots \ldots \ldots \ldots \ldots \ldots \\
\text { (modified), rod } \ldots \ldots \ldots \ldots \ldots \ldots\end{array}$ & ea & 37 & $\begin{array}{l}1265 \\
1266\end{array}$ & $\begin{array}{l}\text { Iron, electrolytic, disk } \ldots \ldots \ldots \ldots \ldots \ldots \\
\text { Set, } 1 \text { ea of } 1261,1262,1263, \\
1264 \text {, and } 1265, \ldots \ldots \ldots \ldots \ldots \ldots \ldots\end{array}$ & $\begin{array}{l}\text { ea } \\
\text { set }\end{array}$ & $\begin{array}{r}49 \\
179\end{array}$ \\
\hline $\begin{array}{r}1099 \\
1101 \\
C 1101\end{array}$ & $\begin{array}{l}\text { Gasometric, lron, electrolytic, rod } \ldots \ldots \ldots \ldots \\
\text { Brass, cartridge B } . \cdots \cdots \cdots \cdots \cdots \cdots \cdots \cdots\end{array}$ & $\begin{array}{l}\text { ea } \\
\text { ea }\end{array}$ & $\begin{array}{l}37 \\
69\end{array}$ & $\begin{array}{l}1301 \\
1302\end{array}$ & $\begin{array}{l}\text { Metal coating, nonmagnetic, } 0.00010 \text { in thick. } \\
\text { Metal coating, nonmagnetic, } 0.00025 \text { in thick. }\end{array}$ & $\begin{array}{l}\text { ea } \\
\text { ea }\end{array}$ & $\begin{array}{l}39 \\
39\end{array}$ \\
\hline $\begin{array}{r}1101 \\
1102 \\
C 1102 \\
1103 \\
C 1103\end{array}$ & 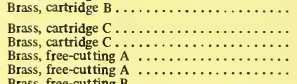 & $\begin{array}{l}\text { ea } \\
\text { ea } \\
\text { ea } \\
\text { ea } \\
\text { ea }\end{array}$ & $\begin{array}{l}69 \\
69 \\
69 \\
69 \\
69\end{array}$ & $\begin{array}{l}1303 \\
1304 \\
1305 \\
1306 \\
1307\end{array}$ & $\begin{array}{l}\text { Metal coating, nonmagnetic, } 0.00050 \text { in thick . } \\
\text { Metal coating, nonmanetic,, } 0.00075 \text { in thick. } \\
\text { Metal coating, nonmagnetic, } 0.0010 \text { in thick } . . \\
\text { Metal coating, nonmagnetic, } 0.0015 \text { in thick .. } \\
\text { Metal coating, nonmagnetic, } 0.0020 \text { in thick.. }\end{array}$ & $\begin{array}{l}\text { ea } \\
\text { ea } \\
\text { ea } \\
\text { ea } \\
\text { ea }\end{array}$ & $\begin{array}{l}39 \\
39 \\
39 \\
39 \\
39\end{array}$ \\
\hline $\begin{array}{r}1104 \\
\mathrm{C} 1104 \\
1105 \\
\mathrm{C} 1105 \\
1106 \\
\mathrm{C} 1106\end{array}$ & 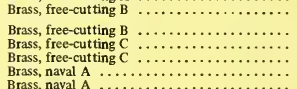 & $\begin{array}{l}\text { ea } \\
\text { ea } \\
\text { ea } \\
\text { ea } \\
\text { ea } \\
\text { ea }\end{array}$ & $\begin{array}{l}69 \\
69 \\
69 \\
69 \\
69 \\
69\end{array}$ & $\begin{array}{l}1308 \\
1309 \\
1310 \\
1311 \\
1312\end{array}$ & $\begin{array}{l}\text { Metal coating, nonmagnetic, } 0.0025 \text { in thick .. } \\
\text { Metal coating, nonmagnetic, } 0.0027 \text { in thick } . . \\
\text { Metal coating, nonmagnetic, } 0.0032 \text { in thick } . . \\
\text { Metal coating, nonmanetic, } 0.0055 \text { in thick .. } \\
\text { Metal coating, nonmagnetic, } 0.0080 \text { in thick } \ldots\end{array}$ & $\begin{array}{l}\text { ea } \\
\text { ea } \\
\text { ea } \\
\text { ea } \\
\text { ea }\end{array}$ & $\begin{array}{r}39 \\
39 \\
39 \\
39 \\
39\end{array}$ \\
\hline C1106 & Brass, naval A $\ldots \ldots \ldots \ldots \ldots \ldots \ldots \ldots \ldots \ldots \ldots \ldots$ & ea & 69 & 1313 & Metal coating, nonmagnetic, 0.010 in thick ... & ea & 39 \\
\hline $\begin{array}{r}1107 \\
\text { C } 1107 \\
1108 \\
\text { C } 1108\end{array}$ & $\begin{array}{l}\text { Brass, naval B } \\
\text { Brass, naval B } \\
\text { Brass, naval C } \\
\text { Bral } \\
\text { Brass, naval C }\end{array}$ & $\begin{array}{l}\text { ea } \\
\text { ea } \\
\text { ea } \\
\text { ea }\end{array}$ & $\begin{array}{l}69 \\
69 \\
69 \\
69\end{array}$ & $\begin{array}{l}1314 \\
1315 \\
1316 \\
1317\end{array}$ & $\begin{array}{l}\text { Metal coating, nonmagnetic, } 0.015 \text { in thick .... } \\
\text { Metal coating, nonmagnetic, } 0.020 \text { in thick } \ldots \\
\text { Metal coating, nonmagnetic, } 0.025 \text { in thick } \ldots \\
\text { Metal coating, nonmagnetic, } 0.03 \text { in thick .... }\end{array}$ & $\begin{array}{l}\text { ea } \\
\text { ea } \\
\text { ea } \\
\text { ea }\end{array}$ & $\begin{array}{l}39 \\
39 \\
39 \\
39\end{array}$ \\
\hline 1109 & 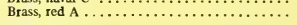 & ea & 69 & 1318 & Metal coating, nonmagnetic, 0.04 in thick .... & ea & 39 \\
\hline $\begin{array}{rl}C & 1109 \\
1110 \\
\text { C } 1110 \\
1111\end{array}$ & 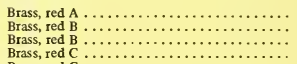 & $\begin{array}{l}\text { ea } \\
\text { ea } \\
\text { ea } \\
\text { ea }\end{array}$ & $\begin{array}{l}69 \\
69 \\
69 \\
69\end{array}$ & $\begin{array}{l}1319 \\
1320 \\
1331 \\
1332\end{array}$ & $\begin{array}{l}\text { Metal coating, nonmagnetic, } 0.06 \text { in thick } \ldots . . \\
\text { Metal coating, nonmagnetic, } 0.08 \text { in thick } \ldots . . \\
\text { Metal coating, magnetic, } 0.00012 \text { in thick } \ldots . . . \\
\text { Metal coating, magnetic, } 0.00035 \text { in thick } \ldots . .\end{array}$ & $\begin{array}{l}\text { ea } \\
\text { ea } \\
\text { ea } \\
\text { ea }\end{array}$ & $\begin{array}{r}39 \\
39 \\
39 \\
39\end{array}$ \\
\hline Ci111 & 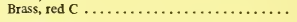 & ea & 69 & 1333 & Metal coating, magnetic, 0.00055 in thick.... & & 39 \\
\hline $\begin{aligned} 1112 \\
\mathrm{C} 1112 \\
1113 \\
\mathrm{C} 1113\end{aligned}$ & 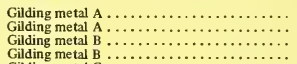 & $\begin{array}{l}\text { ea } \\
\text { ea } \\
\text { ea } \\
\text { ea }\end{array}$ & $\begin{array}{l}69 \\
69 \\
69 \\
69\end{array}$ & $\begin{array}{l}1334 \\
1335 \\
1336 \\
1337\end{array}$ & $\begin{array}{l}\text { Metal coating, magnetic, } 0.00075 \text { in thick ..... } \\
\text { Metal coating, magnetic, } 0.0010 \text { in thick } \ldots . . \\
\text { Metal coating, magnetic, } 0.0013 \text { in thick } \ldots . . . \\
\text { Metal coating, magnetic, } 0.0016 \text { in thick ..... }\end{array}$ & $\begin{array}{l}\text { ea } \\
\text { ea } \\
\text { ea } \\
\text { ea }\end{array}$ & $\begin{array}{l}39 \\
39 \\
39 \\
39\end{array}$ \\
\hline 1114 & Gilding metal $\mathrm{C}$.................................... & ea & 69 & & Metal coating, magnetic, 0.0020 in thick ...... & ea & 39 \\
\hline $\begin{array}{l}\text { C } 1114 \\
1115 \\
\text { C } 1115 \\
1116\end{array}$ & 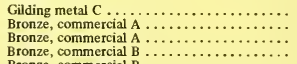 & $\begin{array}{l}\text { ea } \\
\text { ea } \\
\text { ea } \\
\text { ea }\end{array}$ & $\begin{array}{l}69 \\
69 \\
69 \\
69\end{array}$ & $\begin{array}{l}1339 \\
1341 \\
1342 \\
1343\end{array}$ & $\begin{array}{l}\text { Metal coating, magnetic, } 0.0025 \text { in thick } \ldots . . . \\
\text { Metal coating, magnetic, } 0.00012 \text { in thick } \ldots . . . \\
\text { Metal coating, magnetic, } 0.00035 \text { in thick } \ldots . . \\
\text { Metal coating, magnetic, } 0.00065 \text { in thick .... }\end{array}$ & $\begin{array}{l}\text { ea } \\
\text { ea } \\
\text { ea } \\
\text { ea }\end{array}$ & $\begin{array}{l}39 \\
39 \\
39 \\
39\end{array}$ \\
\hline C 1116 & 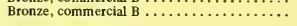 & ea & 69 & 1344 & Metal coating, magnetic, 0.0010 in thick ...... & ea & 39 \\
\hline $\begin{array}{r}1117 \\
\text { C1117 } \\
1118 \\
\text { C } 1118\end{array}$ & 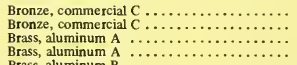 & $\begin{array}{l}\text { ea } \\
\text { ea } \\
\text { ea } \\
\text { ea }\end{array}$ & $\begin{array}{l}69 \\
69 \\
69 \\
69\end{array}$ & $\begin{array}{l}1345 \\
1346 \\
1351 \\
1352\end{array}$ & $\begin{array}{l}\text { Metal coating, magnetic, } 0.0015 \text { in thick } \ldots \ldots \\
\text { Metal coating, magnetic, } 0.0020 \text { in thick } \ldots \ldots . \\
\text { Set of one each } 1307 \text { and } 1311 \ldots \ldots \ldots \ldots \ldots \\
\text { Set of one each } 1332 \text { and } 1334 \ldots \ldots \ldots \ldots \ldots\end{array}$ & $\begin{array}{l}\text { ea } \\
\text { ea } \\
\operatorname{set}(2) \\
\operatorname{set}(2)\end{array}$ & $\begin{array}{l}39 \\
39 \\
51 \\
51\end{array}$ \\
\hline 1119 & Brass, aluminum B.$\ldots \ldots \ldots \ldots \ldots \ldots \ldots \ldots$ & ea & 69 & 1353 & Set of one each 1335 and $1339 \ldots$ & $\operatorname{set}(2)$ & 51 \\
\hline $\begin{array}{r}\mathrm{C} 1119 \\
1120 \\
\mathrm{C} 1120\end{array}$ & $\begin{array}{l}\text { Brass, aluminum B B } \ldots \ldots \ldots \ldots \ldots \ldots \ldots \ldots \ldots \\
\text { Brass, aluminum C C } \ldots \ldots \ldots \ldots \ldots \ldots \ldots \ldots \ldots \ldots \\
\text { Brass, aluminum C. }\end{array}$ & $\begin{array}{l}\text { ea } \\
\text { ea } \\
\text { ea }\end{array}$ & $\begin{array}{l}69 \\
69 \\
69\end{array}$ & $\begin{array}{l}1361 \\
1362\end{array}$ & $\begin{array}{l}\text { Set of one each } 1302,1303,1305 \text {, } \\
\text { and } 1307 \ldots \ldots \ldots 1306,1310,1311 \text {, }\end{array}$ & $\operatorname{set}(4)$ & 75 \\
\hline $\begin{array}{l}C 1120 \\
1121\end{array}$ & $\begin{array}{l}\text { Brass, aluminum } \mathrm{C} \\
\text { Beryllium copper } \mathrm{CA}-17 \ldots \ldots \ldots \ldots \ldots \ldots \ldots \ldots \\
\end{array}$ & $\begin{array}{l}\text { ea } \\
\text { ea }\end{array}$ & $\begin{array}{l}69 \\
69\end{array}$ & 1362 & $\begin{array}{l}\text { Set of one each } 1306,1310,1311, \\
\text { and } 1312, \ldots \ldots \ldots \ldots \ldots \ldots \ldots \ldots\end{array}$ & $\operatorname{set}(4)$ & 75 \\
\hline $\mathrm{C} 1121$ & Beryllium copper CA-172 $\ldots \ldots \ldots \ldots \ldots \ldots \ldots \ldots$ & ea & 69 & 1363 & & & \\
\hline $\begin{array}{r}1122 \\
\mathrm{C} 1122 \\
1123 \\
\mathrm{C} 1123 \\
1131\end{array}$ & $\begin{array}{l}\text { Beryllium copper CA-170 } \ldots \ldots \ldots \ldots \ldots \ldots \\
\text { Beryllium copper CA-170 } \ldots \ldots \ldots \ldots \ldots \ldots \ldots \ldots \\
\text { Beryllium copper CA-175 } \ldots \ldots \ldots \ldots \ldots \ldots \ldots \ldots \ldots \\
\text { Beryllium copper CA-175 } \ldots \ldots \ldots \ldots \ldots \ldots \ldots \ldots \\
\text { Solder (Sn40-Pb60) } \ldots \ldots \ldots \ldots \ldots \ldots \ldots \ldots \ldots\end{array}$ & $\begin{array}{l}\text { ea } \\
\text { ea } \\
\text { ea } \\
\text { ea } \\
\text { ea }\end{array}$ & $\begin{array}{l}69 \\
69 \\
69 \\
69 \\
54\end{array}$ & $\begin{array}{l}1364 \\
1365\end{array}$ & 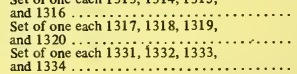 & $\begin{array}{l}\operatorname{set}(4) \\
\operatorname{set}(4) \\
\operatorname{set}(4)\end{array}$ & $\begin{array}{l}75 \\
75 \\
75\end{array}$ \\
\hline
\end{tabular}




\begin{tabular}{|c|c|c|c|c|c|c|c|}
\hline SRM & Type & Unit & Price & SRM & Type & Unit & Price \\
\hline 1366 & Set of one each $1335,1336,1337$, & & & 2001 & $\begin{array}{l}\text { Aluminum on glass, specular spectral } \\
\text { reflectance. }\end{array}$ & & \\
\hline 1367 & Set of one each $1341,1342,1343$, & $\operatorname{set}(4)$ & $\$ 75$ & 2002 & Aluminum on glass, specular spectrai & ea & $\$ 279$ \\
\hline 1368 & 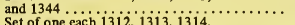 & $\operatorname{set}(4)$ & 75 & & reflectance ................. & ea & 279 \\
\hline 1368 & $\begin{array}{l}\text { Set of one each 1312, 1313,1314, } \\
\text { and } 1315 \ldots \ldots \ldots \ldots \ldots \ldots \ldots \ldots\end{array}$ & $\operatorname{set}(4)$ & 75 & 2003 & 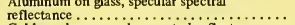 & ea & 279 \\
\hline 1369 & Set of one each $1316,1317,1318$ & & & 2005 & Gold on glass, specular spectral reflectance ... & ea & 279 \\
\hline 1370 & and $1319 \ldots \ldots$ of $1312,1313,1314$, & $\operatorname{set}(4)$ & 75 & $\begin{array}{l}2006 \\
2007\end{array}$ & $\begin{array}{l}\text { Gold on glass, specular spectral reflectance } \ldots . \\
\text { Gold on glass, specular spectral reflectance } \ldots\end{array}$ & $\begin{array}{l}\text { ea } \\
\text { ea }\end{array}$ & $\begin{array}{l}279 \\
279\end{array}$ \\
\hline 1371 & $\begin{array}{l}1315,1316,1317,1318, \text { and } 1319 \ldots \ldots \ldots \\
\text { Gold coating }(\mathrm{Fe}-\mathrm{Ni}-\mathrm{Co}) 30 \text { microinches } \ldots \ldots\end{array}$ & $\begin{array}{l}\text { set }(8) \\
\text { ea }\end{array}$ & $\begin{array}{r}146 \\
70\end{array}$ & & $\begin{array}{l}\text { Gold on glass, specular spectral reflectance ..... } \\
\text { Color std. }\end{array}$ & ea & 279 \\
\hline 1372 & Gold coating (Fo-Ni-Co) 60 microinches ...... & ea & 70 & 2106 & $\begin{array}{l}\text { Color std. } \ldots \ldots \ldots \\
\text { ISCC-NBS color charts } . . . \ldots \ldots \ldots \ldots \ldots\end{array}$ & $\begin{array}{l}\text { set } \\
\text { set }\end{array}$ & $\begin{array}{r}379 \\
9\end{array}$ \\
\hline $\begin{array}{l}1373 \\
1374\end{array}$ & $\begin{array}{l}\text { Gold coating (Fe-Ni-Co) } 120 \text { microinches .... } \\
\text { Gold coating (Fe-Ni-Co) } 280 \text { microinches } . . .\end{array}$ & ea & 70 & 2141 & Urea $\ldots \ldots \ldots \ldots \ldots$ & $2 \mathrm{~g}$ & 37 \\
\hline 1375 & $\begin{array}{l}\text { Gold coating (Fickl-Col) } 30 \text { microinches .... } \\
\text { Gold coating (Nickel) }\end{array}$ & $\begin{array}{l}\text { ea } \\
\text { ea }\end{array}$ & $\begin{array}{l}70 \\
70\end{array}$ & $\begin{array}{l}2142 \\
2143\end{array}$ & $\begin{array}{l}\text { o-Bromobenzoic acid. } \\
\text { p-fluorobenzoic acid. }\end{array}$ & $2 \mathrm{~g}$ & 37 \\
\hline 1376 & Gold coating (Nickel) 60 microinches. & ea & 70 & 2144 & m-chlorobenzoic acid & & \\
\hline $\begin{array}{l}1377 \\
1378\end{array}$ & $\begin{array}{l}\text { Gold coating (Nickel) } 120 \text { microinches } \\
\text { Gold coating (Nickel) } 350 \text { microinches }\end{array}$ & ea & 70 & 2186-I & Potassium dihydrogen phosphate, pD .. & $30 \mathrm{~g}$ & 45 \\
\hline $\begin{array}{l}1378 \\
1381\end{array}$ & $\begin{array}{l}\text { Gold coating (Nickel) } 350 \text { microinches } \\
\text { Set of one each } 1371 \text { and } 1372 \ldots \ldots \ldots\end{array}$ & $\begin{array}{l}\text { ea } \\
\text { set(2) }\end{array}$ & $\begin{array}{r}70 \\
113\end{array}$ & $2186-11$ & Disodium hy drogen phosphate, pD . . . . . . . & $30 \mathrm{~g}$ & 45 \\
\hline 1382 & Set of one each 1372 and $1373 \ldots$. & $\operatorname{set}(2)$ & 113 & $\begin{array}{l}2191 \\
2192\end{array}$ & $\begin{array}{l}\text { Sodium bicarbonate, pD } \\
\text { Sodium carbonate, } \mathrm{pD} . .\end{array}$ & $\begin{array}{l}30 \mathrm{~g} \\
30 \mathrm{~g}\end{array}$ & $\begin{array}{l}45 \\
45\end{array}$ \\
\hline 1383 & Set of one each 1373 and $1374 \ldots .$. & $\operatorname{set}(2)$ & 113 & 2201 & Sodium chloride ion-selective electrode..... & $125 \mathrm{~g}$ & 38 \\
\hline 1384 & Set of one each 1375 and 1376 . & $\operatorname{set}(2)$ & 113 & 2202 & Potassium chloride ion-selective electrode ..... & $160 \mathrm{~g}$ & 38 \\
\hline $\begin{array}{l}1385 \\
1386\end{array}$ & $\begin{array}{l}\text { Set of one each } 1376 \text { and } 1377 \text {. } \\
\text { Set of one each } 1377 \text { and } 1378 \text {. }\end{array}$ & $\begin{array}{l}\operatorname{set}(2) \\
\operatorname{set}(2)\end{array}$ & $\begin{array}{l}113 \\
113\end{array}$ & $\begin{array}{l}2301 \\
2302\end{array}$ & $\begin{array}{l}\text { Gold coating (epoxy) } 30 \text { microinches. } \\
\text { Gold coating (epoxy) } 60 \text { microinches }\end{array}$ & $\begin{array}{l}\text { ea } \\
\text { ea }\end{array}$ & 70 \\
\hline 1398 & Set of one each $1371,1372,1373$ & & & 2303 & $\begin{array}{l}\text { Gold coatung (epoxy) } \\
\text { Gold coating (epoxy) } 120 \text { microinches }\end{array}$ & $\begin{array}{l}\text { ea } \\
\text { ea }\end{array}$ & 70 \\
\hline & $\begin{array}{l}\text { and } 1374 \ldots \ldots \ldots \ldots \ldots \ldots \ldots \\
\text { Set of one each } 1375,1376,1377 \text {, }\end{array}$ & $\operatorname{set}(4)$ & 186 & $\begin{array}{l}2304 \\
2305\end{array}$ & $\begin{array}{l}\text { Gold coating (epoxy) } 280 \text { microinches ....... } \\
\text { Set of one each } 2301 \text { and } 2302 \ldots \ldots \ldots \ldots \ldots\end{array}$ & $\begin{array}{l}\text { ea } \\
\text { set(2) }\end{array}$ & $\begin{array}{r}70 \\
113\end{array}$ \\
\hline 1399 & and $1378 \ldots \ldots \ldots \ldots$ & $\operatorname{set}(4)$ & $\begin{array}{l}186 \\
184\end{array}$ & 2306 & Set of one each 2302 and $2303 \ldots$ & $\operatorname{set}(2)$ & 113 \\
\hline $\begin{array}{l}1402 \\
1403 \\
1404\end{array}$ & $\begin{array}{l}\text { Emittance std., } 1 / 2 \text { in. disk } \ldots \ldots \ldots \\
\text { Emittance std., } 7 / 8 \text { in. disk } \ldots \ldots \ldots \\
\text { Emittance std., } 1 \text { in. disk . . . . . }\end{array}$ & $\begin{array}{l}\text { ea } \\
\text { ea } \\
\text { ea }\end{array}$ & $\begin{array}{l}184 \\
194 \\
209\end{array}$ & $\begin{array}{l}2307 \\
2308\end{array}$ & $\begin{array}{l}\text { Set of one each } 2303 \text { and } 2304 \ldots \ldots \\
\text { Set of one each } 2301,2302,2303, \\
\text { and } 2304 \ldots \ldots \ldots \ldots \ldots \ldots \ldots\end{array}$ & $\begin{array}{l}\operatorname{set}(2) \\
\operatorname{set}(4)\end{array}$ & 113 \\
\hline $\begin{array}{l}1405 \\
1406\end{array}$ & Emittance std., $11 / 8$ in. disk $\ldots .$. & ea & 244 & 2311 & Gold coating (copper) 30 microinches ........... & $\begin{array}{l}\text { set( } 4) \\
\text { ea }\end{array}$ & $\begin{array}{r}186 \\
70\end{array}$ \\
\hline $\begin{array}{l}1406 \\
1407\end{array}$ & $\begin{array}{l}\text { Emittance std., } 11 / 4 \text { in. disk } \ldots \ldots \ldots \ldots \ldots \ldots \\
\text { Emittance std., } 2 \text { in. } \times 2 \text { in. } \ldots \ldots \ldots \ldots \ldots \ldots\end{array}$ & $\begin{array}{l}\text { ea } \\
\text { ea }\end{array}$ & 259 & 2312 & Gold coating (copper) 60 microinches... & ea & 70 \\
\hline 1408 & Emittance std., 1 in. $x 10$ in. $\ldots \ldots \ldots \ldots \ldots, \ldots$ & $\begin{array}{l}\text { ea } \\
\text { ea }\end{array}$ & $\begin{array}{l}394 \\
759\end{array}$ & 2313 & Gold coating (copper) 120 microinches . & ea & 70 \\
\hline 1409 & Emittance std., $3 / 4$ in. $\times 10$ in. ....... & ea & 609 & $\begin{array}{l}2314 \\
2315\end{array}$ & $\begin{array}{l}\text { Gold coating (copper) } 280 \text { microinches....... } \\
\text { Set of one each } 2311 \text { and } 2312 \ldots \ldots \ldots \ldots \ldots\end{array}$ & $\begin{array}{l}\text { ea } \\
\text { set(2) }\end{array}$ & $\begin{array}{r}70 \\
113\end{array}$ \\
\hline 1420 & Emittance std., $1 / 2$ in. disk & ea & 184 & 2316 & Set of one each 2312 and $2313 \ldots .$. & $\operatorname{set}(2)$ & 113 \\
\hline $\begin{array}{l}1421 \\
1422\end{array}$ & Emittance std., $7 / 8$ in. disk $\ldots \ldots$ & ea & $\begin{array}{l}184 \\
184\end{array}$ & 2317 & Set of one each 2313 and 2314 . & $\operatorname{set}(2)$ & 113 \\
\hline $\begin{array}{l}1422 \\
1423\end{array}$ & $\begin{array}{l}\text { Emittance std., } 1 \text { in. disk } \ldots \ldots \ldots \ldots \\
\text { Emittance std., } 1 \text { 1/8 in. disk } \ldots . . . .\end{array}$ & $\begin{array}{l}\text { ea } \\
\text { ea }\end{array}$ & $\begin{array}{l}184 \\
184\end{array}$ & 2318 & $\begin{array}{l}\text { Set of one each } 2311,2312,2313 \text {, and } \\
2314\end{array}$ & $\operatorname{set}(4)$ & \\
\hline 1424 & Emittance std., 1 1/4 in. disk $\ldots \ldots \ldots \ldots \ldots \ldots$ & ea & 184 & 2331 & Tin coating 60 microinches & ea & 70 \\
\hline 1425 & Emittance std., 2 in. $\times 2$ in. $\ldots \ldots \ldots \ldots \ldots$ & ea & 184 & 2332 & Tin coating 110 microinches $\ldots \ldots \ldots \ldots \ldots$ & ea & 70 \\
\hline $\begin{array}{l}1427 \\
1428\end{array}$ & Emittance std., $3 / 4$ in. $\times 10$ in. $\ldots \ldots \ldots \ldots \ldots$ & ea & $\begin{array}{l}184 \\
184\end{array}$ & $\begin{array}{l}2533 \\
2334\end{array}$ & $\begin{array}{l}\text { Tin coating } 160 \text { microinches } \ldots \ldots \ldots \ldots \ldots \ldots \\
\text { Tin coating } 275 \text { microinches }\end{array}$ & ea & 70 \\
\hline $\begin{array}{l}1448 \\
1440\end{array}$ & $\begin{array}{l}\text { Emittance std., } 1 / 4 \text { in. } \times 8 \text { in. } \ldots \ldots \ldots \ldots \ldots \ldots \\
\text { Emittance std., } 1 / 2 \text { in. disk ....................... }\end{array}$ & $\begin{array}{l}\text { ea } \\
\text { ea }\end{array}$ & 184 & $\begin{array}{l}2354 \\
2335\end{array}$ & 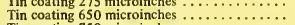 & $\begin{array}{l}\text { ea } \\
\text { ea }\end{array}$ & $\begin{array}{l}70 \\
70\end{array}$ \\
\hline 1441 & Emittance std., $7 / 8$ in. disk ............ & ea & 184 & 2336 & Tin coating 750 microinches $\ldots \ldots \ldots . .$. & ea & 70 \\
\hline $\begin{array}{l}1442 \\
1443\end{array}$ & Emittance std., 1 in. disk ........ & ea & 184 & 2338 & Set of one each 2332 and $2335 \ldots \ldots \ldots$ & $\operatorname{set}(2)$ & 113 \\
\hline $\begin{array}{l}1443 \\
1444\end{array}$ & $\begin{array}{ll}\text { Emittance std., } 1 & 1 / 8 \text { in. disk } \\
\text { Emittance std., } 1 & 1 / 4 \text { in. disk } \ldots\end{array}$ & ea & 184 & 2339 & Set of one each 2331, 2333, 2334, & & \\
\hline 1445 & $\begin{array}{l}\text { Emittance std., } 11 / 4 \text { in. disk } \ldots \ldots \ldots \ldots \ldots \ldots \\
\text { Emittance std., } 2 \text { in. } \times 2 \text { in. } \ldots \ldots \ldots \ldots \ldots \ldots\end{array}$ & $\begin{array}{l}\text { ea } \\
\text { ea }\end{array}$ & $\begin{array}{l}184 \\
184\end{array}$ & 2340 & $\begin{array}{l}\text { and } 2336 \ldots \ldots 3733 \\
\text { Set of one each } 2331,23\end{array}$ & set(4) & 186 \\
\hline 1475 & $\begin{array}{l}\text { Emittance stene, linear } \ldots \ldots \ldots \ldots \ldots \ldots \ldots \ldots \ldots \\
\text { Polyethyla }\end{array}$ & $50 \mathrm{~g}$ & 104 & 2340 & 2334,2335 , and $2336 \ldots \ldots \ldots \ldots$ & $\operatorname{set}(6)$ & 265 \\
\hline 1476 & Polyethylene, branched $\ldots \ldots \ldots \ldots \ldots \ldots$ & $50 \mathrm{~g}$ & 79 & 3200 & Tape, magnetic, secondary std. . ....... & ea & 699 \\
\hline $\begin{array}{l}1511 \\
1512\end{array}$ & $\begin{array}{l}\text { Cyclohexane - dielectric } \ldots \ldots \ldots \ldots \ldots \ldots \\
1,2 \text { Dichloroethane dielectric }\end{array}$ & $\begin{array}{l}400 \mathrm{ml} \\
400 \mathrm{ml}\end{array}$ & $\begin{array}{l}129 \\
124\end{array}$ & $\begin{array}{l}4200-B \\
4201-B\end{array}$ & $\begin{array}{l}\text { Cesium-137, gamma-ray point source } \\
\text { Niobium- } 94 \text {, gamma-ray point source }\end{array}$ & ea & 64 \\
\hline 1513 & Nitrobenzene dielectric $\ldots \ldots \ldots \ldots \ldots \ldots \ldots, \ldots$ & $400 \mathrm{ml}$ & 124 & 4202 & $\begin{array}{l}\text { Niobium-94, gamma-ray point source } \ldots \ldots \ldots \\
\text { Cadmium-109, gamma-ray point source } \ldots . . .\end{array}$ & $\begin{array}{c}\text { ea } \\
\text { ea }\end{array}$ & 156 \\
\hline 1516 & Permittivity Std., $38 \mathrm{~mm} \times 2.5 \mathrm{~mm}$............. & ea & 197 & 4203 & Cobalt-60, gamma-ray point source .......... & ea & 97 \\
\hline $\begin{array}{l}1517 \\
1518\end{array}$ & Permittivity Std., $38 \mathrm{~mm} \times 5 \mathrm{~mm} \ldots$ & ea & 197 & 4205 & Thorium- 228 , gamma-ray point source...$\ldots$ & ea & 102 \\
\hline $\begin{array}{l}1518 \\
1519\end{array}$ & $\begin{array}{l}\text { Permittivity Std., } 51 \mathrm{~mm} \times 2.5 \mathrm{~mm} \ldots \ldots \ldots \ldots \\
\text { Permittivity Std., } 51 \mathrm{~mm} \times 5 \mathrm{~mm} \ldots \ldots \ldots \ldots \ldots\end{array}$ & ea & 197 & 4206 & Thorium-228, gamma-ray point source $\ldots \ldots$. & ea & 102 \\
\hline 1541 & & $\begin{array}{l}\text { ea } \\
\text { ea }\end{array}$ & 197 & 4207 & Cesium- $137, \mathrm{~g}$ & ea & 64 \\
\hline 1571 & $\begin{array}{l}\text { Mossbauer, iron foil } \\
\text { Botanical, orchard leaves, trace element ........ }\end{array}$ & $\begin{array}{l}\text { ea } \\
75\end{array}$ & $\begin{array}{r}154 \\
72\end{array}$ & 4210 & point source. & ea & \\
\hline \multirow{3}{*}{$\begin{array}{l}1573 \\
1577 \\
1578 \\
1579 \\
1601\end{array}$} & & & & $\begin{array}{l}4211 \\
4212\end{array}$ & $\begin{array}{l}\text { Americium-241, gamma-ray point source } \ldots \ldots \\
\text { Krypton- } 85 \text {, gamma-ray point source } \ldots . . .\end{array}$ & $\begin{array}{l}\text { ea } \\
\text { ea }\end{array}$ & $\begin{array}{l}132 \\
164\end{array}$ \\
\hline & \multirow{2}{*}{$\begin{array}{l}\text { Botanical, tomato leaves } \ldots \ldots \ldots \ldots \ldots \ldots \ldots \\
\text { Biological, Liver, bovine } \ldots \ldots \ldots \ldots \ldots \ldots \ldots \ldots \\
\text { Biological, Tuna, albacore } \ldots \ldots \ldots \ldots \ldots \ldots \ldots \ldots \\
\text { Powdered lead-base paint } \ldots \ldots \ldots \ldots \ldots \ldots \ldots \\
\text { Carbon dioxide in nitrogen, } 308 \text { ppm } \ldots \ldots \ldots \ldots\end{array}$} & $50 \mathrm{~g}$ & 92 & 4213 & \multirow{3}{*}{ 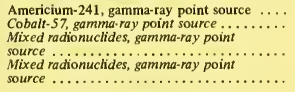 } & \multirow[t]{3}{*}{ ea } & \multirow[t]{3}{*}{132} \\
\hline & & ${ }_{\mathrm{cyl}}^{35 \mathrm{~g}}$ & $\begin{array}{r}35 \\
154\end{array}$ & $\begin{array}{l}4214 \\
4215\end{array}$ & & & \\
\hline \multirow{4}{*}{$\begin{array}{l}1602 \\
1603 \\
1604 a \\
1605 \\
1606\end{array}$} & \multirow{4}{*}{$\begin{array}{l}\text { Carbon dioxide in nitrogen, } 346 \mathrm{ppm} \ldots \ldots \ldots \\
\text { Carbon dioxide in nitrogen, } 384 \mathrm{ppm} \ldots \ldots \ldots \ldots \\
\text { Oxygcn in nitrogen, } 1.5 \mathrm{ppm} \ldots \ldots \ldots \ldots \ldots \ldots \\
\text { Oxygen in nitrogen, } 10 \mathrm{ppm} \ldots \ldots \ldots \ldots \ldots \ldots \\
\text { Oxygen in nitrogen, } 112 \mathrm{ppm} \ldots \ldots \ldots \ldots \ldots \ldots\end{array}$} & cyl & $\begin{array}{l}154 \\
154\end{array}$ & 4216 & & & \\
\hline & & cyl & 114 & 4222 & \multirow{4}{*}{ 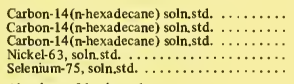 } & \multirow{4}{*}{$\begin{array}{r}3 \mathrm{~g} \\
3 \mathrm{~g} \\
3 \mathrm{~g} \\
4 \mathrm{~g} \\
4.6 \mathrm{~g}\end{array}$} & 59 \\
\hline & & cyl & 114 & 4223 & & & 59 \\
\hline & & cyl & 114 & ${ }_{4224}$ & & & $\begin{array}{r}59 \\
153\end{array}$ \\
\hline $\begin{array}{l}1607 \\
1608\end{array}$ & $\begin{array}{l}\text { Oxygen in nitrogen, } 211 \mathrm{ppm} \ldots \ldots \ldots \ldots \ldots \\
\text { Oxygen in nitrogen, } 978 \mathrm{ppm} \ldots \ldots \ldots \ldots \ldots\end{array}$ & $\begin{array}{l}\text { cyl } \\
\text { cyl }\end{array}$ & $\begin{array}{l}114 \\
114\end{array}$ & $\begin{array}{l}4226 \\
4228\end{array}$ & & & $\begin{array}{l}153 \\
122\end{array}$ \\
\hline $\begin{array}{l}1608 \\
1609\end{array}$ & $\begin{array}{l}\text { Oxygen in nitrogen, } 978 \mathrm{ppm} \ldots \ldots \ldots \ldots \ldots \\
\text { Oxygen in nitrogen, } 20.98 \mathrm{~mole} \text { percent } \ldots \ldots \ldots\end{array}$ & cyl & 114 & 4229 & Aluminum-26, soln. std. $\ldots \ldots \ldots \ldots \ldots \ldots$ & $4.6 \mathrm{~g}$ & 204 \\
\hline 1610 & Hydrocarbon in air, 0.103 mole percent $\ldots . .$. & cyl & 178 & 4230 & Chromium- 51 , soln std. . . & & \\
\hline 1611 & Hydrocarbon in air, 0.0107 mole percent $\ldots .$. & cyl & 178 & $\begin{array}{l}4231 \\
4232\end{array}$ & $\begin{array}{l}\text { Cobalt-56, soln, std. } \ldots \ldots \ldots \ldots \ldots \ldots \ldots \ldots \\
\text { Silver-110m,soln, std. } \ldots \ldots \ldots \ldots \ldots \ldots \ldots \ldots\end{array}$ & & \\
\hline $\begin{array}{l}1613 \\
1621\end{array}$ & $\begin{array}{l}\text { Hydrocarbon in air, } 0.000102 \text { mole percent } \ldots \\
\text { Sulfur in residual fuel oil, } 1.05 \text { wt percent } \ldots\end{array}$ & $\begin{array}{l}\text { cyl } \\
100 \mathrm{ml}\end{array}$ & $\begin{array}{r}178 \\
34\end{array}$ & 4233 & Cesium-137.Barium. $137 m$, soln. std. .......... & & \\
\hline 1622 & Sulfur in residual fuel oil, 2.14 wt percent $\ldots$ & $100 \mathrm{ml}$ & 34 & 4234 & Barium. 140-Lanthanum-140, soln. std. . . . . & & \\
\hline $\begin{array}{l}1623 \\
1624\end{array}$ & $\begin{array}{l}\text { Sulfur in residual fuel oil, } 0.268 \text { wt percent } \ldots \\
\text { Sulfur in distillate fuel oil, } 0.211 \text { wt percent } \ldots\end{array}$ & $\begin{array}{l}100 \mathrm{ml} \\
100 \mathrm{ml}\end{array}$ & $\begin{array}{l}34 \\
34\end{array}$ & $\begin{array}{l}4235 \\
4236\end{array}$ & $\begin{array}{l}\text { Krypton-85, gamma-ray g } \\
\text { Xenon-133, gas std. ..... }\end{array}$ & ea & 104 \\
\hline 1625 & Sulfur dioxide permeation tube $10 \mathrm{~cm} \ldots \ldots$. & ea & 65 & $\begin{array}{l}4240 \\
4242-\mathrm{B}\end{array}$ & 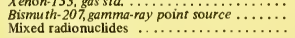 & $450 \mathrm{ml}$ & 54 \\
\hline 1626 & Sulfur dioxide permeation tube $5 \mathrm{~cm}$.. & & 65 & $4243-\mathrm{B}$ & $\begin{array}{l}\text { Mixed radionuclides } \\
\text { Mixed radionuclides }\end{array}$ & & 54 \\
\hline $\begin{array}{l}1627 \\
1630\end{array}$ & $\begin{array}{l}\text { Sulfur dioxide permeation tube } 2 \mathrm{~cm} . \\
\text { Trace mercury in coal } \ldots \ldots \ldots \ldots \ldots \ldots\end{array}$ & $\begin{array}{l}\text { ea } \\
50 \mathrm{~g}\end{array}$ & $\begin{array}{l}65 \\
49\end{array}$ & 4244-B B & 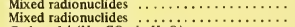 & $\begin{array}{l}50 \mathrm{ml} \\
15 \mathrm{ml}\end{array}$ & 54 \\
\hline 1631 & Sulfur in coal, three concentrations, & & & 4245 & Carbon. $14\left(\mathrm{Na}_{2} \mathrm{CO}_{3}\right.$ in $\left.\mathrm{H}_{2} \mathrm{O}\right)$ & & \\
\hline 1651 & $\begin{array}{l}5 \text { sets of } 3 \ldots \ldots \ldots \ldots \ldots \ldots \ldots \ldots \\
\text { Zirconium-barium chromatc heat source }\end{array}$ & set & 57 & $\begin{array}{l}4246 \\
4247\end{array}$ & $\begin{array}{l}\text { Carbon. } 14\left(\mathrm{Na}_{2} \mathrm{CO}_{3} \text { in } \mathrm{H}_{2}^{2} \mathrm{O}\right) \ldots \ldots \ldots \ldots \ldots \ldots \\
\text { Carbon } 14\left(\mathrm{Na}_{2} \mathrm{CO}_{3} \text { in } \mathrm{H}_{2} \mathrm{O}\right) \ldots \ldots \ldots \ldots \ldots \ldots\end{array}$ & & \\
\hline 1651 & powder $(\mathrm{ca} 350 \mathrm{cal} / \mathrm{g}) \ldots \ldots \ldots \ldots \ldots \ldots$ & $50 \mathrm{~g}$ & 59 & 4252 & Mixed radionuclides, test std. & $450 \mathrm{ml}$ & 50 \\
\hline 1652 & $\begin{array}{l}\text { Zirconiumbarium chromate heat source } \\
\text { powder }(\mathrm{ca} 390 \mathrm{cal} / \mathrm{g} \text { ) }\end{array}$ & 50 & 59 & $\begin{array}{l}4253 \\
4300\end{array}$ & $\begin{array}{l}\text { tuclides, test std. } \\
\text { s std. .......... }\end{array}$ & & 50 \\
\hline 1653 & $\begin{array}{l}\text { powder (ca } 390 \text { cal } / \mathrm{g}) \text {.................. } \\
\text { Zirconiumbarium chromate heat source }\end{array}$ & $50 \mathrm{~g}$ & $3=$ & 4301 & 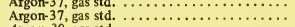 & $10 \mathrm{ml}$ & 72 \\
\hline & powder $(\mathrm{ca} 425 \mathrm{cal} / \mathrm{g}) \ldots \ldots \ldots \ldots \ldots$ & $50 \mathrm{~g}$ & 59 & 4302 & Argon 39 , gas std. .. & & \\
\hline 1654 & $\begin{array}{l}\alpha \text {-Quartz for hydrofluoric acid } \\
\text { solution cal orimetry ............ }\end{array}$ & & 179 & $\begin{array}{l}4303 \\
4304\end{array}$ & $\begin{array}{l}\text { Argon. } 39, \text { gas std. . } 1 \ldots . . \\
\text { Xenon-131m, gas sid. . }\end{array}$ & & \\
\hline 1810 & Linerboard for tape test & pkg & 37 & 4305 & 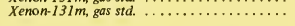 & & \\
\hline & & & & & & & \\
\hline
\end{tabular}




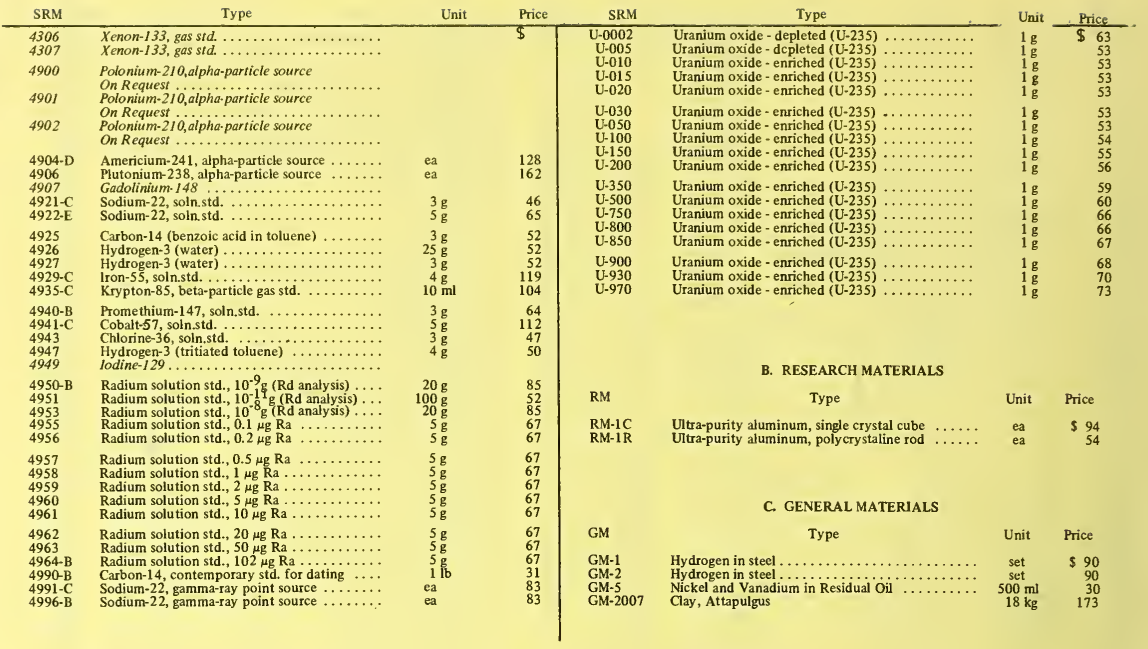




\section{Statistical Abstract of the United States}

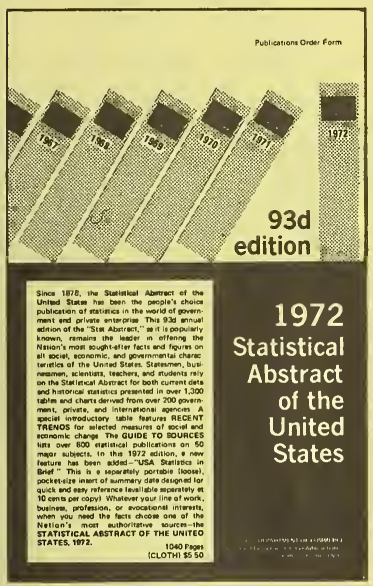

Since 1878, the Statistical Abstract of the United States has been the people's choice publication of statistics in the world of government and private enterprise. This 93d annual edition of the "Stat Abstract," as it is popularly known, remains the leader in offering the Nation's most sought-after facts and figures on all social, economic, and governmental characteristics of the United States. Statesmen, businessmen, scientists, teachers, and students rely on the Statistical Abstract for both current data and historical statistics presented in over 1,300 tables and charts derived from over 200 government, private, and international agencies. A special introductory table features RECENT TRENDS for selected measures of social and economic change. The GUIDE TO SOURCES lists over 800 statistical publications on 50 major subjects. In this 1972 edition, a new feature has been added-"USA Statistics in Brief." This is a separately portable (loose), pocket-size insert of summary data designed for quick and easy reference (available separately at 10 cents per copy). Whatever your line of work, business, profession, or avocational interests, when you need the facts choose one of the Nation's most authoritative sources-the STATISTICAL ABSTRACT OF THE UNITED STATES, 1972.

1040 Pages

(CLOTH) $\$ 5.50$

(please detach along this dotted line)

(please type or print)

ORDER FORM

Plaase send me copy(les) of

1972 Statistical Abstract of the United States at $\$ 5.50$

\begin{tabular}{lll}
\hline Name & & \\
\hline Address & State code \\
\hline City & Zip con ORDER PAYABLE
\end{tabular}

MAKE CHECK OR MONEY ORDER PAYABLE TO SUPERINTENDENT OF DOCUMENTS

To Insure Prompt. Accurate Shipment, Place Correct Address on Mailing Label Below

U.S. Government Printing Office Public Documents Department Washington, D.C. 20402

OFFICIAL BUSINESS

Return after 5 days
Name

Streat Address

City, State, and ZIP Code (check, money order, or Supt. of Documents

coupons) or charge to my Deposit Account No.

Total Amount

MAIL ORDER FORM WITH PAYMENT TO

Superintendent of Documents

U.S. Government Printing Office Washington, D.C. 20402

or any U.S. Department of Commerce field office

\begin{tabular}{|c|}
\hline 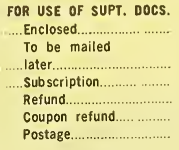 \\
\hline
\end{tabular}

S

$$
\begin{gathered}
\text { POSTAGE AND FEES } \\
\text { PAID } \\
\text { U.S. GOVERNMENT } \\
\text { PRINTING OFFICE } \\
375 \\
\text { SPecIal Fourth Class Rata } \\
\text { Book }
\end{gathered}
$$


